\title{
Radiative neutrino masses, lepton flavor mixing and muon $g-2$ in a leptoquark model
}

\section{Di Zhang}

Institute of High Energy Physics, Chinese Academy of Sciences, Beijing 100049, China

School of Physical Sciences, University of Chinese Academy of Sciences, Beijing 100049, China

E-mail: zhangdi@ihep.ac.cn

ABstract: We propose a leptoquark model with two scalar leptoquarks $S_{1}\left(\overline{3}, 1, \frac{1}{3}\right)$ and $\widetilde{R}_{2}\left(3,2, \frac{1}{6}\right)$ to give a combined explanation of neutrino masses, lepton flavor mixing and the anomaly of muon $g-2$, satisfying the constraints from the radiative decays of charged leptons. The neutrino masses are generated via one-loop corrections resulting from a mixing between $S_{1}$ and $\widetilde{R}_{2}$. With a set of specific textures for the leptoquark Yukawa coupling matrices, the neutrino mass matrix possesses an approximate $\mu-\tau$ reflection symmetry with $\left(M_{\nu}\right)_{e e}=0$ only in favor of the normal neutrino mass ordering. We show that this model can successfully explain the anomaly of muon $g-2$ and current experimental neutrino oscillation data under the constraints from the radiative decays of charged leptons.

Keywords: Neutrino Physics, Beyond Standard Model

ArXiv ePrint: 2105.08670 


\section{Contents}

1 Introduction 1

2 The LQ model 3

2.1 Radiative neutrino masses 4

2.2 LFV decays and muon $g-2 \quad 5$

3 Neutrino masses and lepton flavor mixing $\quad 9$

4 Numerical calculations $\quad 13$

$\begin{array}{llr}5 & \text { Summary } & 18\end{array}$

\section{Introduction}

As a quantum field theory with $\mathrm{SU}(3)_{\mathrm{c}} \times \mathrm{SU}(2)_{\mathrm{L}} \times \mathrm{U}(1)_{\mathrm{Y}}$ gauge symmetry, the Standard Model (SM) has successfully described the behaviors of strong, weak and electromagnetic interactions of all the known fundamental particles. However, there exist some compelling evidences that the SM is incomplete and only an effective field theory at low energy scales at least in its leptonic flavor sector [1]. In the neutrino sector, a number of successful neutrino oscillation experiments have firmly proved that neutrinos have tiny masses and the lepton flavor mixing exists [2]. These facts are in contradiction with the SM and strongly call for new physics beyond the SM. Usually, some new heavy particles are introduced to generate the tiny neutrino masses at the tree level, such as right-handed neutrinos, $\mathrm{SU}(2)_{\mathrm{L}}$-triplet Higgs and $\mathrm{SU}(2)_{\mathrm{L}}$-triplet fermions in the type-I [3-7], type-II [8-13] and type-III $[14,15]$ seesaw mechanisms, respectively. An alternative and interesting way to naturally generate the tiny neutrino masses is via radiative corrections [16-18] (see. e.g., refs. $[19,20]$, for a recent review). In the charged-lepton sector, there is a longstanding anomaly of the muon anomalous magnetic moment, namely the discrepancy between the theoretical computations and its measured value, which motivates extensions of the SM with new couplings to leptons. Very recently, the Muon $(g-2)$ Collaboration at Fermi National Laboratory has announced their new result [21], which combined with the previous result from the E821 experiment at Brookhaven National Laboratory [22], leads to a $4.2 \sigma$ discrepancy with the SM prediction [23], that is $\Delta a_{\mu}=a_{\mu}(\operatorname{Exp})-a_{\mu}(\mathrm{SM})=(251 \pm 59) \times$ $10^{-11}$ with $1 \sigma$ error. ${ }^{1}$ Inspired by this new result, there have been plenty of theoretical works gushing out to explain this discrepancy [25-88]. A systematic summary of various new-physics scenarios and a great deal of earlier references can be found in refs. [60, 89].

\footnotetext{
${ }^{1}$ Note that the recent result from lattice QCD is compatible with the experimental value [24].
} 
Motivated by above facts, we attempt to extend the SM to give a combined explanation of the tiny neutrino masses, lepton flavor mixing and muon $g-2$. Unfortunately, the most popular way to generate neutrino masses via the three typical seesaw mechanisms can not account for the anomaly of muon $g-2[71,90]$. Thus in this work, we focus on an interesting scenario of radiative neutrino masses, where two scalar leptoquarks (LQs), $S_{1}\left(\overline{3}, 1, \frac{1}{3}\right)$ and $\widetilde{R}_{2}\left(3,2, \frac{1}{6}\right)$ with the numbers in parenthesis denoting $\mathrm{SU}(3)_{\mathrm{c}} \times \mathrm{SU}(2)_{\mathrm{L}} \times \mathrm{U}(1)_{\mathrm{Y}}$ quantum numbers are introduced into the SM. LQ extensions of the SM have been discussed extensively due to its possible origination from the grand unification framework [91-94] and its ability to explain neutrino masses via radiative corrections, the muon $g-2$ and lepton flavor universality violations in semi-leptonic $B$-meson decays $[26,31,39,60,69,70,72,82,87,95$ 121] (see, e.g., ref. [122], for a recent review of LQs). In this work, we only focus on the combined explanation of neutrino masses, lepton flavor mixing and the anomaly of muon $g-2$, as well as constraints from the lepton-flavor-violation (LFV) radiative decays of charged leptons, by means of $S_{1}$ and $\widetilde{R}_{2}$. It will show that our model works well and differs from the previous works at least in following three aspects:

- To our best knowledge, it is the first time that the combination of $S_{1}$ and $\widetilde{R}_{2}$ is used to simultaneously interpret the tiny neutrino masses and the anomaly of muon $g-2$, including the constraints from radiative decays of charged leptons, though the radiative neutrino masses generated by $S_{1}$ and $\widetilde{R}_{2}$ and contributions from $S_{1}$ to the muon $g-2$ have been separately discussed before.

- It is well-known that the mixing between $S_{1}$ and $\widetilde{R}_{2}$ induced by a LQ-Higgs interaction plays a greatly important role in radiatively generating neutrino masses [100, 101]. This mixing also has significant effects on the branching ratios of radiative chargedlepton decays and then the muon $g-2$. Thus we recalculate all contributions from the physical $S_{1}$ and $\widetilde{R}_{2}$ to the branching ratios of radiative charged-lepton decays and the muon $g-2$ in this work, after the mixing between non-physical $S_{1}$ and $\widetilde{R}_{2}$ is absorbed into the redefinitions of physical fields.

- Motivated by the current experimental data for neutrino oscillations, a set of specific structures for the leptoquark Yukawa coupling matrices are taken to make the neutrino mass matrix approximately possess the $\mu-\tau$ reflection symmetry [123, 124]. Given the Yukawa coupling matrices in eq. (3.1), the neutrino mass matrix additionally has the texture with one zero element, i.e., $\left(M_{\nu}\right)_{e e}=0$, which rules out the inverted neutrino mass ordering, and in the limit of $\mu-\tau$ reflection symmetry leads to the lightest neutrino mass $m_{1}$ determined fully by values of $\theta_{12}$ and $\theta_{13}$, besides $\theta_{23}=45^{\circ}, \delta_{\mathrm{CP}}= \pm 90^{\circ}$ and $\rho, \sigma=0$ or $90^{\circ}$. We analytically discussed the corresponding neutrino masses and lepton flavor mixing induced by this novel neutrino mass matrix with both the exact and approximate $\mu-\tau$ reflection symmetry in detail.

The rest of this paper is organized as follows. In section 2, we construct the LQ model with $S_{1}$ and $\widetilde{R}_{2}$, and calculate the radiative neutrino mass matrix at the one-loop level, the branching ratios of radiative decays of charged leptons and the muon anomalous magnetic 
moment. With specific textures of the leptoquark Yukawa coupling matrices, the neutrino masses and lepton flavor mixing with an approximate $\mu-\tau$ reflection symmetry are discussed in detail in section 3. The numerical calculations are carried out in section 4. Finally, we make a summary in section 5 .

\section{The LQ model}

Enforcing baryon number conservation (e.g., assigning baryon number $B=-\frac{1}{3}$ to $S_{1}$ and $\widetilde{R}_{2}^{*}$ under a global $\mathrm{U}(1)_{\mathrm{B}}$ symmetry) to avoid potentially dangerous proton decay, ${ }^{2}$ the Lagrangian associated with $S_{1}\left(\overline{3}, 1, \frac{1}{3}\right)$ and $\widetilde{R}_{2}\left(3,2, \frac{1}{6}\right)$ is given by

$$
\begin{aligned}
\mathcal{L}_{\mathrm{LQ}}= & \lambda_{i \alpha}^{\mathrm{L}} \overline{Q_{i \mathrm{~L}}^{c}} \epsilon \ell_{\alpha \mathrm{L}} S_{1}+\lambda_{i \alpha}^{\mathrm{R}} \overline{u_{i \mathrm{R}}^{c}} E_{\alpha \mathrm{R}} S_{1}+\lambda_{i \alpha} \overline{d_{i \mathrm{R}}} \widetilde{R}_{2}^{T} \epsilon \ell_{\alpha \mathrm{L}}+\text { h.c. }, \\
& +\left(D_{\mu} S_{1}\right)^{\dagger}\left(D^{\mu} S_{1}\right)+\left(D_{\mu} \widetilde{R}_{2}\right)^{\dagger}\left(D^{\mu} \widetilde{R}_{2}\right)-V_{\mathrm{LQ}},
\end{aligned}
$$

where as usual, $Q_{\mathrm{L}}$ and $\ell_{\mathrm{L}}$ are the left-handed quark and lepton doublets of $\mathrm{SU}(2)_{\mathrm{L}}$, and $u_{\mathrm{R}}, d_{\mathrm{R}}$ and $E_{\mathrm{R}}$ are the right-handed up-type quark, down-type quark and charged lepton singlets of $\mathrm{SU}(2)_{\mathrm{L}}$, and the Latin and Greek subscripts running over $(1,2,3)$ and $(e, \mu, \tau)$ are quark and lepton flavor indices, respectively. In eq. (2.1), $\epsilon$ is the $\mathrm{SU}(2)_{\mathrm{L}}$ Levi-Civita tensor, the superscript " $c$ " stands for charge conjugation (i.e., $\psi^{c}=C \bar{\psi}^{T}$ with $C$ being the charge-conjugation matrix), $D_{\mu}$ is the gauge covariant derivative. The most general renormalizable potential involving $S_{1}$ and $\widetilde{R}_{2}$ is

$$
\begin{aligned}
V_{\mathrm{LQ}}= & \mu_{S}^{2} S_{1}^{\dagger} S_{1}+\mu_{R}^{2} \widetilde{R}_{2}^{\dagger} \widetilde{R}_{2}+\left(\lambda_{\mathrm{mix}} S_{1}^{*} \widetilde{R}_{2}^{\dagger} H+\text { h.c. }\right)+\lambda_{H S}\left(H^{\dagger} H\right)\left(S_{1}^{\dagger} S_{1}\right)+\lambda_{H R}^{(1)}\left(H^{\dagger} H\right)\left(\widetilde{R}_{2}^{\dagger} \widetilde{R}_{2}\right) \\
& +\lambda_{H R}^{(3)}\left(H^{\dagger} \tau^{I} H\right)\left(\widetilde{R}_{2}^{\dagger} \tau^{I} \widetilde{R}_{2}\right)+\lambda_{S}\left(S_{1}^{\dagger} S_{1}\right)^{2}+\lambda_{R}^{(1)}\left(\widetilde{R}_{2}^{\dagger} \widetilde{R}_{2}\right)^{2}+\lambda_{R}^{(8)}\left(\widetilde{R}_{2}^{\dagger} T^{A} \widetilde{R}_{2}\right)^{2} \\
& +\lambda_{S R}^{(1)}\left(S_{1}^{\dagger} S_{1}\right)\left(\widetilde{R}_{2}^{\dagger} \widetilde{R}_{2}\right)+\lambda_{S R}^{(8)}\left(S_{1}^{\dagger} T^{A} S_{1}\right)\left(\widetilde{R}_{2}^{\dagger} T^{A} \widetilde{R}_{2}\right)
\end{aligned}
$$

in which $H$ is the SM Higgs doublet, and $\tau^{I}$ (for $I=1,2,3$ ) and $T^{A}$ (for $A=1, \ldots, 8$ ) are the Pauli and Gell-Mann matrices, respectively. As we will see later, the LQ-Higgs interaction $\lambda_{\text {mix }} S_{1}^{*} \widetilde{R}_{2}^{\dagger} H$ provides a mixing between $S_{1}$ and $\widetilde{R}_{2}^{-\frac{1}{3} *}$ after spontaneous gauge symmetry breaking (SSB), which is required to realize lepton number violation and hence radiatively generate neutrino masses. After the neutral Higgs field obtains its vacuum expectation value, i.e., $\left\langle H^{0}\right\rangle=v / \sqrt{2}$, the mass matrix for $S_{1}$ and $\widetilde{R}_{2}^{-\frac{1}{3} *}$ in the basis $\left(S_{1}, \widetilde{R}_{2}^{-\frac{1}{3} *}\right)$ is found to be

$$
M_{\text {mix }}^{2}=\left(\begin{array}{cc}
m_{S}^{2} & \frac{v}{\sqrt{2}} \lambda_{\text {mix }} \\
\frac{v}{\sqrt{2}} \lambda_{\text {mix }}^{*} & m_{R}^{2}
\end{array}\right)
$$

\footnotetext{
${ }^{2}$ At the renomalizable level, the baryon-number-violating diquark couplings (i.e., $\overline{Q_{i \mathrm{~L}}^{c}} \epsilon Q_{j \mathrm{~L}} S_{1}^{\dagger}$ and $\overline{u_{i \mathrm{R}}^{c}} d_{j \mathrm{R}} S_{1}^{\dagger}$ with $i, j$ being flavor indices) are forbidden by the global $\mathrm{U}(1)_{\mathrm{B}}$ symmetry. Note that the dimension five operators involving the leptoquark $\widetilde{R}_{2}$ (i.e., $\epsilon_{a b e} u_{i \mathrm{R}}^{a} d_{j \mathrm{R}}^{b}\left(H^{\dagger} \widetilde{R}_{2}^{e}\right) / \Lambda$ and $\epsilon_{a b e} u_{i \mathrm{R}}^{a} e_{\alpha \mathrm{R}}\left(\widetilde{R}_{2}^{b} \epsilon \widetilde{R}_{2}^{e}\right) / \Lambda$ with $i, j, \alpha$ and $a, b, e$ being flavor indices and color indices, respectively) also lead to proton decay, but such dimension five operators can be forbidden by embedding the SM in a larger gauge symmetry group with the right symmetry properties [125, 126].
} 


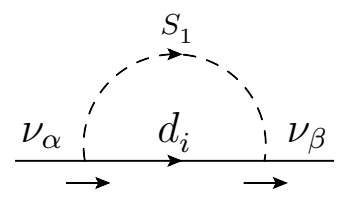

(a)

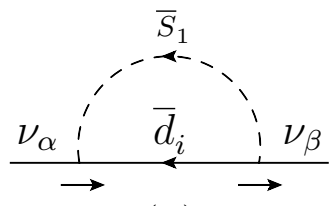

(b)

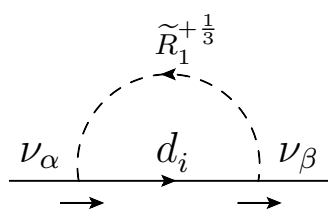

(c)

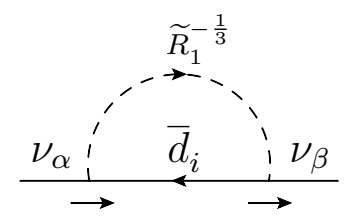

(d)

Figure 1. Self-energy diagrams for Majorana neutrinos. The arrows below vertices represent the orientations of fermion flow [127, 128].

with

$$
m_{S}^{2}=\mu_{S}^{2}+\frac{v^{2}}{2} \lambda_{H S}, \quad m_{R}^{2}=\mu_{R}^{2}+\frac{v^{2}}{2}\left(\lambda_{H R}^{(1)}+\lambda_{H R}^{(3)}\right) .
$$

For simplicity, we take all parameters in eqs. (2.3) and (2.4) to be real, thus we can make the following transformations

$$
S_{1} \rightarrow \cos \theta S_{1}+\sin \theta \widetilde{R}_{2}^{-\frac{1}{3} *}, \quad \widetilde{R}_{2}^{-\frac{1}{3} *} \rightarrow-\sin \theta S_{1}+\cos \theta \widetilde{R}_{2}^{-\frac{1}{3} *}
$$

with $\tan 2 \theta=\sqrt{2} \lambda_{\text {mix }} v /\left(m_{R}^{2}-m_{S}^{2}\right)$ to work in the basis of the physical LQs which are named in the same way as the non-physical leptoquarks. To avoid misunderstandings, we will make it clear when referring to the physical fields. The masses of the physical LQs are given by

$$
M_{1,2}^{2}=\frac{1}{2}\left[m_{S}^{2}+m_{R}^{2} \pm \sqrt{\left(m_{S}^{2}-m_{R}^{2}\right)^{2}+2 \lambda_{\text {mix }}^{2} v^{2}}\right], \quad M_{3}^{2}=\mu_{R}^{2}+\frac{v^{2}}{2}\left(\lambda_{H R}^{(1)}-\lambda_{H R}^{(3)}\right),
$$

where $M_{1}, M_{2}$ and $M_{3}$ are the masses of the physical $S_{1}, \widetilde{R}_{2}^{-\frac{1}{3}}$ and $\widetilde{R}_{2}^{+\frac{2}{3}}$, respectively.

Given the transformations in eq. (2.5), the Yukawa couplings involving the physical LQs after SSB are

$$
\begin{aligned}
\mathcal{L}_{\mathrm{Y}}= & \overline{\nu_{\alpha}}\left(\lambda_{i \alpha}^{*} \sin \theta P_{\mathrm{R}}-\lambda_{i \alpha}^{\mathrm{L}} \cos \theta P_{\mathrm{L}}\right) d_{i} S_{1}+\overline{l_{\alpha}^{c}}\left(\lambda_{i \alpha}^{\mathrm{L}} P_{\mathrm{L}}+\lambda_{i \alpha}^{\mathrm{R}} P_{\mathrm{R}}\right) \cos \theta u_{i} S_{1} \\
& -\overline{\nu_{\alpha}}\left(\lambda_{i \alpha}^{*} \cos \theta P_{\mathrm{R}}+\lambda_{i \alpha}^{\mathrm{L}} \sin \theta P_{\mathrm{L}}\right) d_{i} \widetilde{R}_{2}^{-\frac{1}{3} *}+\overline{l_{\alpha}^{c}}\left(\lambda_{i \alpha}^{\prime \mathrm{L}} P_{\mathrm{L}}+\lambda_{i \alpha}^{\mathrm{R}} P_{\mathrm{R}}\right) \sin \theta u_{i} \widetilde{R}_{2}^{-\frac{1}{3} *} \\
& +\lambda_{i \alpha} \overline{d_{i}} P_{\mathrm{L}} l_{\alpha} \widetilde{R}_{2}^{+\frac{2}{3}}+\text { h.c. },
\end{aligned}
$$

with $\lambda^{\prime \mathrm{L}}=V^{T} \lambda^{\mathrm{L}}$, where we work in the down-type quark and charged-lepton mass eigenstate bases (i.e., $d_{i}$ and $l_{\alpha}$ ), and the up-type quark fields have been transformed into their mass eigenstates $u_{i}$ by the Cabibbo-Kobayashi-Maskawa (CKM) matrix $V$.

\subsection{Radiative neutrino masses}

In the considered LQ model described by eqs. (2.1) and (2.2), neutrinos are exactly massless at the tree level, but they can acquire masses radiatively via the one loop diagrams in figure $1 .^{3}$ The general structure of the Majorana neutrino self-energy is given by [130]

$$
\Sigma_{\alpha \beta}(\not p)=\not p P_{\mathrm{L}} \Sigma_{\alpha \beta}^{\mathrm{R} *}\left(p^{2}\right)+\not p P_{\mathrm{R}} \Sigma_{\alpha \beta}^{\mathrm{R}}\left(p^{2}\right)+P_{\mathrm{L}} \Sigma_{\alpha \beta}^{\mathrm{M} *}\left(p^{2}\right)+P_{\mathrm{R}} \Sigma_{\alpha \beta}^{\mathrm{M}}\left(p^{2}\right),
$$

\footnotetext{
${ }^{3}$ All Feynman diagrams in this work are produced by using JaxoDraw [129].
} 
where only the last two terms will contribute to neutrino masses. From the Feynman diagrams in figure 1 , one can obtain

$$
\begin{aligned}
-\mathrm{i} \Sigma_{\alpha \beta}(\not p)= & N_{c} \int \frac{\mathrm{d}^{4} k}{(2 \pi)^{4}}\left[\left(\lambda_{i \beta}^{*} \sin \theta P_{\mathrm{R}}-\lambda_{i \beta}^{\mathrm{L}} \cos \theta P_{\mathrm{L}}\right) \frac{1}{\not k-\hat{m}_{i}}\left(\lambda_{i \alpha} \sin \theta P_{\mathrm{L}}-\lambda_{i \alpha}^{\mathrm{L} *} \cos \theta P_{\mathrm{R}}\right)\right. \\
& \times \frac{1}{(k-p)^{2}-M_{1}^{2}}+\left(\lambda_{i \beta} \sin \theta P_{\mathrm{L}}-\lambda_{i \beta}^{\mathrm{L} *} \cos \theta P_{\mathrm{R}}\right) \frac{1}{\not k-\hat{m}_{i}}\left(\lambda_{i \alpha}^{*} \sin \theta P_{\mathrm{R}}-\lambda_{i \alpha}^{\mathrm{L}} \cos \theta P_{\mathrm{L}}\right) \\
& \times \frac{1}{(k-p)^{2}-M_{1}^{2}}+\left(\lambda_{i \beta}^{*} \cos \theta P_{\mathrm{R}}-\lambda_{i \beta}^{\mathrm{L}} \sin \theta P_{\mathrm{L}}\right) \frac{1}{\not k-\hat{m}_{i}}\left(\lambda_{i \alpha} \cos \theta P_{\mathrm{L}}-\lambda_{i \alpha}^{\mathrm{L} *} \sin \theta P_{\mathrm{R}}\right) \\
& \times \frac{1}{(k-p)^{2}-M_{2}^{2}}+\left(\lambda_{i \beta} \cos \theta P_{\mathrm{L}}-\lambda_{i \beta}^{\mathrm{L} *} \sin \theta P_{\mathrm{R}}\right) \frac{1}{\not k-\hat{m}_{i}}\left(\lambda_{i \alpha}^{*} \cos \theta P_{\mathrm{R}}-\lambda_{i \alpha}^{\mathrm{L}} \sin \theta P_{\mathrm{L}}\right) \\
& \left.\times \frac{1}{(k-p)^{2}-M_{2}^{2}}\right],
\end{aligned}
$$

then radiative neutrino mass matrix $M_{\nu}$ can be extracted from eq. (2.9), that is ${ }^{4}$

$$
\begin{aligned}
\left(M_{\nu}\right)_{\alpha \beta}=\Sigma_{\alpha \beta}^{\mathrm{M}}(0)= & \frac{\mathrm{i}}{2} N_{c}\left(\lambda_{i \alpha}^{*} \lambda_{i \beta}^{\mathrm{L} *}+\lambda_{i \alpha}^{\mathrm{L} *} \lambda_{i \beta}^{*}\right) \sin 2 \theta \int \frac{\mathrm{d}^{4} k}{(2 \pi)^{4}} \frac{\hat{m}_{i}}{k^{2}-\hat{m}_{i}^{2}} \\
& \times\left[\frac{1}{(k-p)^{2}-M_{2}^{2}}-\frac{1}{(k-p)^{2}-M_{1}^{2}}\right] \\
= & -\frac{N_{c}}{2(4 \pi)^{2}}\left(\lambda_{i \alpha}^{*} \lambda_{i \beta}^{\mathrm{L} *}+\lambda_{i \alpha}^{\mathrm{L} *} \lambda_{i \beta}^{*}\right) \hat{m}_{i} \sin 2 \theta\left[\frac{M_{2}^{2} \ln \frac{\hat{m}_{i}^{2}}{M_{2}^{2}}}{M_{2}^{2}-\hat{m}_{i}^{2}}-\frac{M_{1}^{2} \ln \frac{\hat{m}_{i}^{2}}{M_{1}^{2}}}{M_{1}^{2}-\hat{m}_{i}^{2}}\right] \\
\simeq & \frac{3 \sin 2 \theta}{32 \pi^{2}} \ln \frac{M_{2}^{2}}{M_{1}^{2}}\left[\left(\lambda^{\dagger}\right)_{\alpha i} \hat{m}_{i}\left(\lambda^{\mathrm{L} *}\right)_{i \beta}+\left(\lambda^{\mathrm{L} \dagger}\right)_{\alpha i} \hat{m}_{i}\left(\lambda^{*}\right)_{i \beta}\right],
\end{aligned}
$$

where $N_{c}=3$ is the color number and $\hat{m}=\left(m_{\mathrm{d}}, m_{\mathrm{s}}, m_{\mathrm{b}}\right)$ denotes the masses of down-type quarks. Note that the mixing between $S_{1}$ and $\widetilde{R}_{2}^{-\frac{1}{3}}$ is important for radiatively generating neutrino masses, and if the masses of the physical $S_{1}$ and $\widetilde{R}_{2}^{-\frac{1}{3}}$ are exactly degenerate, three neutrinos will remain massless at the one-loop level.

\subsection{LFV decays and muon $g-2$}

Induced by the Yukawa couplings of the physical LQs in eq. (2.7), the LFV decays of charged leptons, i.e., $l_{\alpha}^{-} \rightarrow l_{\beta}^{-}+\gamma$ with $(\alpha, \beta)=(\tau, \mu),(\tau, e)$ and $(\mu, e)$ occur at the oneloop level, as shown in figure 2. The muon anomalous magnetic moment can be easily extracted from the total amplitude of the muon radiative decay. Note that all physical LQs will contribute to the total amplitude and lepton anomalous magnetic moment, but the dominant contributions only come from the physical $S_{1}$ and $\widetilde{R}_{2}^{-\frac{1}{3}}$ due to the chiral enhancement.

\footnotetext{
${ }^{4}$ All one-loop integrals in this work are calculated with the help of Package-X [131, 132].
} 


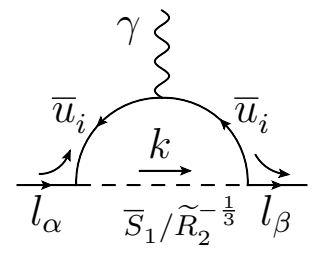

(a)

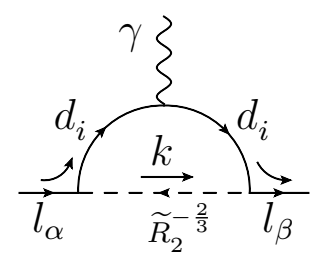

(e)

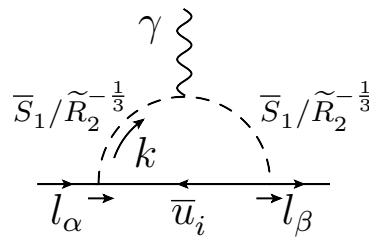

(b)

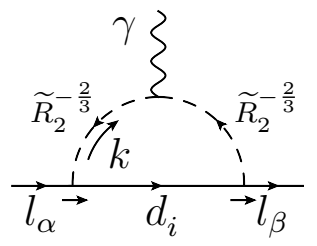

(f)

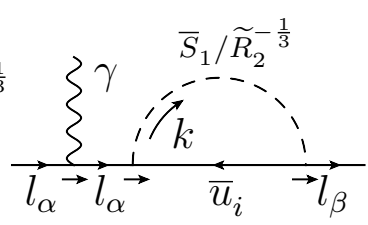

(c)

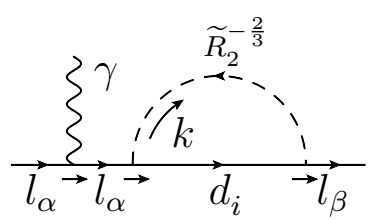

(g)

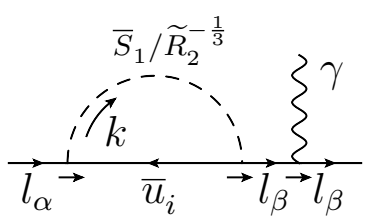

(d)

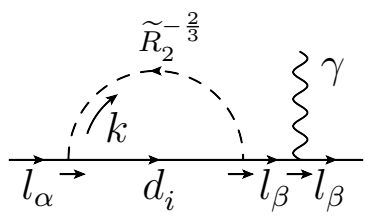

(h)

Figure 2. Diagrams for the radiative decays of charged leptons, i.e., $l_{\alpha}^{-} \rightarrow l_{\beta}^{-}+\gamma$ with $(\alpha, \beta)=(\tau, \mu),(\tau, e)$ and $(\mu, e)$. The arrows around vertices represent the orientations of fermion flow $[127,128]$.

The amplitudes for the radiative decays of charged leptons mediated by the physical $S_{1}$ as shown by diagrams (a) - (d) in figure 2 are given by

$$
\begin{aligned}
\mathrm{i} \mathcal{M}_{(\mathrm{a})}^{S_{1}}= & \frac{2}{3} N_{c} e \cos ^{2} \theta \epsilon_{\mu}^{*}(q) \bar{u}(p-q)\left\{\int \frac{\mathrm{d}^{4} k}{(2 \pi)^{4}}\left(\lambda_{i \beta}^{\mathrm{L} *} P_{\mathrm{R}}+\lambda_{i \beta}^{\mathrm{R} *} P_{\mathrm{L}}\right) \frac{\left(\not p-\not k-\not q+\tilde{m}_{i}\right)}{(k+q-p)^{2}-\tilde{m}_{i}^{2}}\right. \\
& \left.\times \gamma^{\mu} \frac{\left(\not p-\not k+\tilde{m}_{i}\right)}{(k-p)^{2}-\tilde{m}_{i}^{2}}\left(\lambda_{i \alpha}^{\mathrm{L}} P_{\mathrm{L}}+\lambda_{i \alpha}^{\mathrm{R}} P_{\mathrm{R}}\right) \frac{1}{k^{2}-M_{1}^{2}}\right\} u(p), \\
\mathrm{i} \mathcal{M}_{(\mathrm{b})}^{S_{1}}= & \frac{1}{3} N_{c} e \cos ^{2} \theta \epsilon_{\mu}^{*}(q) \bar{u}(p-q)\left\{\int \frac{\mathrm{d}^{4} k}{(2 \pi)^{4}}\left(\lambda_{i \beta}^{\prime \mathrm{L} *} P_{\mathrm{R}}+\lambda_{i \beta}^{\mathrm{R} *} P_{\mathrm{L}}\right) \frac{\left(\not p-\not k+\tilde{m}_{i}\right)}{(k-p)^{2}-\tilde{m}_{i}^{2}}\right. \\
& \left.\times\left(\lambda_{i \alpha}^{\prime \mathrm{L}} P_{\mathrm{L}}+\lambda_{i \alpha}^{\mathrm{R}} P_{\mathrm{R}}\right) \frac{1}{(k-q)^{2}-M_{1}^{2}}(2 k-q)^{\mu} \frac{1}{k^{2}-M_{1}^{2}}\right\} u(p), \\
\mathrm{i} \mathcal{M}_{(\mathrm{c})}^{S_{1}}= & -\frac{N_{c} e \cos ^{2} \theta}{m_{\alpha}^{2}-m_{\beta}^{2}} \epsilon_{\mu}^{*}(q) \bar{u}(p-q)\left\{\int \frac{\mathrm{d}^{4} k}{(2 \pi)^{4}}\left(\lambda_{i \beta}^{\prime \mathrm{L} *} P_{\mathrm{R}}+\lambda_{i \beta}^{\mathrm{R} *} P_{\mathrm{L}}\right) \frac{\left(\not p-\not k-\not p+\tilde{m}_{i}\right)}{(k+q-p)^{2}-\tilde{m}_{i}^{2}}\right. \\
& \left.\times\left(\lambda_{i \alpha}^{\prime \mathrm{L}} P_{\mathrm{L}}+\lambda_{i \alpha}^{\mathrm{R}} P_{\mathrm{R}}\right)\left(\not p-\not q+m_{\alpha}\right) \gamma^{\mu} \frac{1}{k^{2}-M_{1}^{2}}\right\} u(p), \\
\mathrm{i} \mathcal{M}_{(\mathrm{d})}^{S_{1}}= & \frac{N_{c} e \cos ^{2} \theta}{m_{\alpha}^{2}-m_{\beta}^{2}} \epsilon_{\mu}^{*}(q) \bar{u}(p-q)\left\{\int \frac{\mathrm{d}^{4} k}{(2 \pi)^{4}} \gamma^{\mu}\left(\not p+m_{\beta}\right)\left(\lambda_{i \beta}^{\prime \mathrm{L} *} P_{\mathrm{R}}+\lambda_{i \beta}^{\mathrm{R} *} P_{\mathrm{L}}\right)\right. \\
& \left.\times \frac{\left(\not p-\not k+\tilde{m}_{i}\right)}{(k-p)^{2}-\tilde{m}_{i}^{2}}\left(\lambda_{i \alpha}^{\prime \mathrm{L}} P_{\mathrm{L}}+\lambda_{i \alpha}^{\mathrm{R}} P_{\mathrm{R}}\right) \frac{1}{k^{2}-M_{1}^{2}}\right\} u(p),
\end{aligned}
$$


in which $\tilde{m}=\left(m_{\mathrm{u}}, m_{\mathrm{c}}, m_{\mathrm{t}}\right)$ and $m=\left(m_{e}, m_{\mu}, m_{\tau}\right)$ denote the masses of up-type quarks and charged leptons, respectively. Then the total amplitude mediated by the physical $S_{1}$ is found to be

$$
\mathrm{i} \mathcal{M}^{S_{1}}=-\frac{\mathrm{i} e}{(4 \pi)^{2}} \epsilon_{\mu}^{*}(q) \bar{u}(p-q)\left(\mathcal{A}_{\mathrm{L}}^{S_{1}} P_{\mathrm{L}}+\mathcal{A}_{\mathrm{R}}^{S_{1}} P_{\mathrm{R}}\right) \mathrm{i} \sigma^{\mu \nu} q_{\nu} u(p),
$$

where

$$
\begin{aligned}
& \mathcal{A}_{\mathrm{L}}^{S_{1}}=\frac{N_{c} \cos ^{2} \theta}{12 M_{1}^{2}}\left[2 \tilde{m}_{i} \lambda_{i \alpha}^{\mathrm{L}} \lambda_{i \beta}^{\mathrm{R} *} \mathcal{F}\left(\frac{\tilde{m}_{i}^{2}}{M_{1}^{2}}\right)-\left(m_{\beta} \lambda_{i \alpha}^{\prime \mathrm{L}} \lambda_{i \beta}^{\prime \mathrm{L} *}+m_{\alpha} \lambda_{i \alpha}^{\mathrm{R}} \lambda_{i \beta}^{\mathrm{R} *}\right) \mathcal{G}\left(\frac{\tilde{m}_{i}^{2}}{M_{1}^{2}}\right)\right], \\
& \mathcal{A}_{\mathrm{R}}^{S_{1}}=\frac{N_{c} \cos ^{2} \theta}{12 M_{1}^{2}}\left[2 \tilde{m}_{i} \lambda_{i \alpha}^{\mathrm{R}} \lambda_{i \beta}^{\prime \mathrm{L} *} \mathcal{F}\left(\frac{\tilde{m}_{i}^{2}}{M_{1}^{2}}\right)-\left(m_{\alpha} \lambda_{i \alpha}^{\prime \mathrm{L}} \lambda_{i \beta}^{\prime \mathrm{L} *}+m_{\beta} \lambda_{i \alpha}^{\mathrm{R}} \lambda_{i \beta}^{\mathrm{R} *}\right) \mathcal{G}\left(\frac{\tilde{m}_{i}^{2}}{M_{1}^{2}}\right)\right],
\end{aligned}
$$

with

$$
\mathcal{F}(x)=\frac{7-8 x+x^{2}+2(2+x) \ln x}{(1-x)^{3}}, \quad \mathcal{G}(x)=\frac{1+4 x-5 x^{2}+2 x(2+x) \ln x}{(1-x)^{4}} .
$$

If $x \ll 1$ is satisfied, $\mathcal{F}(x)$ and $\mathcal{G}(x)$ may be expanded into $\mathcal{F}(x) \simeq 7+4 \ln x$ and $\mathcal{G}(x) \simeq 1$ at the leading order, respectively.

The contributions from the physical $\widetilde{R}_{2}^{-\frac{1}{3}}$ can be easily obtained by making the replacements $\cos ^{2} \theta \rightarrow \sin ^{2} \theta$ and $M_{1} \rightarrow M_{2}$ in eqs. (2.12)-(2.13), that is

$$
\mathrm{i} \mathcal{M}^{\widetilde{R}_{2}^{-\frac{1}{3}}}=\mathrm{i} \mathcal{M}^{S_{1}}\left(M_{1} \rightarrow M_{2}\right) \tan ^{2} \theta,
$$

with $\mathcal{A}_{\mathrm{L}}^{\widetilde{R}_{2}^{-\frac{1}{3}}}=\mathcal{A}_{\mathrm{L}}^{S_{1}}\left(M_{1} \rightarrow M_{2}\right) \tan ^{2} \theta$ and $\mathcal{A}_{\mathrm{R}}^{\widetilde{R}^{-\frac{1}{3}}}=\mathcal{A}_{\mathrm{R}}^{S_{1}}\left(M_{1} \rightarrow M_{2}\right) \tan ^{2} \theta$.

The contributions from $\widetilde{R}_{2}^{+\frac{2}{3}}$ can be achieved by calculating diagrams (e)-(h) in figure 2, namely

$\mathrm{i} \mathcal{M}_{(\mathrm{e})}^{\widetilde{R}_{2}^{+\frac{2}{3}}}=\frac{1}{3} N_{c} e \lambda_{i \alpha} \lambda_{i \beta}^{*} \epsilon_{\mu}^{*}(q) \bar{u}(p-q)\left\{\int \frac{\mathrm{d}^{4} k}{(2 \pi)^{4}} P_{\mathrm{R}} \frac{\not p-\not k-\not p+\hat{m}_{i}}{(k+q-p)^{2}-\hat{m}_{i}^{2}} \gamma^{\mu} \frac{\not p-\not k+\hat{m}_{i}}{(k-p)^{2}-\hat{m}_{i}^{2}} P_{\mathrm{L}} \frac{1}{k^{2}-M_{3}^{2}}\right\} u(p)$, $\mathrm{i} \mathcal{M}_{(\mathrm{f})}^{\widetilde{R}_{2}^{+\frac{2}{3}}}=\frac{2}{3} N_{c} e \lambda_{i \alpha} \lambda_{i \beta}^{*} \epsilon_{\mu}^{*}(q) \bar{u}(p-q)\left\{\int \frac{\mathrm{d}^{4} k}{(2 \pi)^{4}} P_{\mathrm{R}} \frac{\not p-\not k+\hat{m}_{i}}{(k-p)^{2}-\hat{m}_{i}^{2}} P_{\mathrm{L}} \frac{1}{(k-q)^{2}-M_{3}^{2}} \frac{1}{k^{2}-M_{3}^{2}}\right\} u(p)$, $\mathrm{i}_{\mathcal{M}_{(\mathrm{g})}}^{\widetilde{R}_{2}^{+\frac{2}{3}}}=-\frac{N_{c} e \lambda_{i \alpha} \lambda_{i \beta}^{*}}{m_{\alpha}^{2}-m_{\beta}^{2}} \epsilon_{\mu}^{*}(q) \bar{u}(p-q)\left\{\int \frac{\mathrm{d}^{4} k}{(2 \pi)^{4}} P_{\mathrm{R}} \frac{\not p-\not k-\not q+\hat{m}_{i}}{(k+q-p)^{2}-\hat{m}_{i}^{2}} P_{\mathrm{L}}\left(\not p-\not p+m_{\alpha}\right) \gamma^{\mu} \frac{1}{k^{2}-M_{3}^{2}}\right\} u(p)$, $\mathrm{i} \mathcal{M}_{(\mathrm{h})}^{\widetilde{R}_{2}^{+\frac{2}{3}}}=\frac{N_{c} e \lambda_{i \alpha} \lambda_{i \beta}^{*}}{m_{\alpha}^{2}-m_{\beta}^{2}} \epsilon_{\mu}^{*}(q) \bar{u}(p-q)\left\{\int \frac{\mathrm{d}^{4} k}{(2 \pi)^{4}} \gamma^{\mu}\left(\not p+m_{\beta}\right) P_{\mathrm{R}} \frac{\not p-\not k+\hat{m}_{i}}{(k-p)^{2}-\hat{m}_{i}^{2}} P_{\mathrm{L}} \frac{1}{k^{2}-M_{3}^{2}}\right\} u(p)$,

then the total amplitude mediated by $\widetilde{R}_{2}^{+\frac{2}{3}}$ is given by

$$
\mathrm{i} \mathcal{M}^{\widetilde{R}_{2}^{+\frac{2}{3}}}=-\frac{\mathrm{i} e}{(4 \pi)^{2}} \epsilon_{\mu}^{*}(q) \bar{u}(p-q)\left(\mathcal{A}_{\mathrm{L}}^{\widetilde{R}_{2}^{+\frac{2}{3}}} P_{\mathrm{L}}+\mathcal{A}_{\mathrm{R}}^{\widetilde{R}^{+\frac{2}{3}}} P_{\mathrm{R}}\right) \mathrm{i} \sigma^{\mu \nu} q_{\nu} u(p),
$$

where

$$
\mathcal{A}_{\mathrm{L}}^{\widetilde{R}_{2}^{+\frac{2}{3}}}=-\frac{N_{c} m_{\beta}}{12 M_{3}^{2}} \lambda_{i \alpha} \lambda_{i \beta}^{*} \mathcal{I}\left(\frac{\hat{m}_{i}^{2}}{M_{3}^{2}}\right), \quad \mathcal{A}_{\mathrm{R}}^{\widetilde{R}_{2}^{+\frac{2}{3}}}=-\frac{N_{c} m_{\alpha}}{12 M_{3}^{2}} \lambda_{i \alpha} \lambda_{i \beta}^{*} \mathcal{I}\left(\frac{\hat{m}_{i}^{2}}{M_{3}^{2}}\right)
$$


with

$$
\mathcal{I}(x)=\frac{x\left[5-4 x-x^{2}+(2+4 x) \ln x\right]}{(1-x)^{4}},
$$

and $\mathcal{I}(x) \simeq x(5+2 \ln x)$ for $x \ll 1$.

Therefore, the total amplitude for the radiative decays of charged leptons is found to be

$$
\mathrm{i} \mathcal{M}=-\frac{\mathrm{i} e}{(4 \pi)^{2}} \epsilon_{\mu}^{*}(q) \bar{u}(p-q)\left(\mathcal{A}_{\mathrm{L}} P_{\mathrm{L}}+\mathcal{A}_{\mathrm{R}} P_{\mathrm{R}}\right) \mathrm{i} \sigma^{\mu \nu} q_{\nu} u(p)
$$

with

$$
\mathcal{A}_{\mathrm{L}}=\mathcal{A}_{\mathrm{L}}^{S_{1}}+\mathcal{A}_{\mathrm{L}}^{\widetilde{R}_{2}^{-\frac{1}{3}}}+\mathcal{A}_{\mathrm{L}}^{\widetilde{R}_{2}^{+\frac{2}{3}}}, \quad \mathcal{A}_{\mathrm{R}}=\mathcal{A}_{\mathrm{R}}^{S_{1}}+\mathcal{A}_{\mathrm{R}}^{\widetilde{R}_{2}^{-\frac{1}{3}}}+\mathcal{A}_{\mathrm{R}}^{\widetilde{R}^{+\frac{2}{3}}} .
$$

Note that all divergences in the amplitude have been cancelled out, which must be satisfied since there is no corresponding counterterm for charged-lepton radiative decays. With the help of eq. (2.20), one can obtain the branching ratio of $l_{\alpha}^{-} \rightarrow l_{\beta}^{-}+\gamma$,

$$
\mathcal{B}\left(l_{\alpha}^{-} \rightarrow l_{\beta}^{-}+\gamma\right)=\frac{\alpha_{\mathrm{em}}\left(m_{\alpha}^{2}-m_{\beta}^{2}\right)^{3}}{4(4 \pi)^{4} m_{\alpha}^{3} \Gamma_{\alpha}}\left(\left|\mathcal{A}_{\mathrm{L}}\right|^{2}+\left|\mathcal{A}_{\mathrm{R}}\right|^{2}\right)
$$

with $\alpha_{\mathrm{em}}=e^{2} /(4 \pi)$ and $\Gamma_{\alpha}$ being the fine-structure constant and the total decay width of the charged lepton $l_{\alpha}^{-}$, respectively. Then the contributions from the LQs to the muon $g-2$ can be easily extracted from eq. (2.20), i.e.,

$$
\Delta a_{\mu}=\Delta a_{\mu}^{S_{1}}+\Delta a_{\mu}^{\widetilde{R}_{2}^{-\frac{1}{3}}}+\Delta a_{\mu}^{\widetilde{R}_{2}^{+\frac{2}{3}}},
$$

with

$$
\begin{aligned}
\Delta a_{\mu}^{S_{1}} & =-\frac{N_{c} m_{\mu} \cos ^{2} \theta}{6(4 \pi)^{2} M_{1}^{2}}\left[2 \tilde{m}_{i} \operatorname{Re}\left(\lambda_{i \mu}^{\prime \mathrm{L}} \lambda_{i \mu}^{\mathrm{R} *}\right) \mathcal{F}\left(\frac{\tilde{m}_{i}^{2}}{M_{1}^{2}}\right)-m_{\mu}\left(\left|\lambda_{i \mu}^{\prime \mathrm{L}}\right|^{2}+\left|\lambda_{i \mu}^{\mathrm{R}}\right|^{2}\right) \mathcal{G}\left(\frac{\tilde{m}_{i}^{2}}{M_{1}^{2}}\right)\right], \\
\Delta a_{\mu}^{\widetilde{R}_{2}^{-\frac{1}{3}}} & =-\frac{N_{c} m_{\mu} \sin ^{2} \theta}{6(4 \pi)^{2} M_{2}^{2}}\left[2 \tilde{m}_{i} \operatorname{Re}\left(\lambda_{i \mu}^{\mathrm{L}} \lambda_{i \mu}^{\mathrm{R} *}\right) \mathcal{F}\left(\frac{\tilde{m}_{i}^{2}}{M_{2}^{2}}\right)-m_{\mu}\left(\left|\lambda_{i \mu}^{\prime \mathrm{L}}\right|^{2}+\left|\lambda_{i \mu}^{\mathrm{R}}\right|^{2}\right) \mathcal{G}\left(\frac{\tilde{m}_{i}^{2}}{M_{2}^{2}}\right)\right], \\
\Delta a_{\mu}^{\widetilde{R}^{+\frac{2}{3}}} & =\frac{N_{c} m_{\mu}^{2}\left|\lambda_{i \mu}\right|^{2}}{6(4 \pi)^{2} M_{3}^{2}} \mathcal{I}\left(\frac{\hat{m}_{i}^{2}}{M_{3}^{2}}\right) .
\end{aligned}
$$

Considering $\tilde{m}_{i}, \hat{m}_{j} \ll M_{k}$ for $i, j, k=1,2,3$ and $m_{\beta} \ll m_{\alpha}$ for $(\alpha, \beta)=(\tau, \mu),(\tau, e)$, $(\mu, e)$, the branching ratio in eq. (2.22) and the muon $g-2$ in eq. (2.23) are approximately given by

$$
\begin{aligned}
\mathcal{B}\left(l_{\alpha}^{-} \rightarrow l_{\beta}^{-}+\gamma\right) \simeq & \frac{\alpha_{\mathrm{em}} m_{\alpha}^{3}}{16(4 \pi)^{4} \Gamma_{\alpha}}\left\{\left|\tilde{m}_{i} \lambda_{i \alpha}^{\prime \mathrm{L}} \lambda_{i \beta}^{\mathrm{R} *}\left[\frac{\cos ^{2} \theta}{M_{1}^{2}}\left(7+4 \ln \frac{\tilde{m}_{i}^{2}}{M_{1}^{2}}\right)+\frac{\sin ^{2} \theta}{M_{2}^{2}}\left(7+4 \ln \frac{\tilde{m}_{i}^{2}}{M_{2}^{2}}\right)\right]\right|^{2}\right. \\
& \left.+\left|\tilde{m}_{i} \lambda_{i \alpha}^{\mathrm{R}} \lambda_{i \beta}^{\prime \mathrm{L} *}\left[\frac{\cos ^{2} \theta}{M_{1}^{2}}\left(7+4 \ln \frac{\tilde{m}_{i}^{2}}{M_{1}^{2}}\right)+\frac{\sin ^{2} \theta}{M_{2}^{2}}\left(7+4 \ln \frac{\tilde{m}_{i}^{2}}{M_{2}^{2}}\right)\right]\right|^{2}\right\}, \quad(2.25)
\end{aligned}
$$


and

$$
\Delta a_{\mu} \simeq \frac{4 m_{\mu} \tilde{m}_{i}}{(4 \pi)^{2}} \operatorname{Re}\left(\lambda_{i \mu}^{\mathrm{L}} \lambda_{i \mu}^{\mathrm{R} *}\right)\left[\frac{\cos ^{2} \theta}{M_{1}^{2}}\left(\ln \frac{M_{1}^{2}}{\tilde{m}_{i}^{2}}-\frac{7}{4}\right)+\frac{\sin ^{2} \theta}{M_{2}^{2}}\left(\ln \frac{M_{2}^{2}}{\tilde{m}_{i}^{2}}-\frac{7}{4}\right)\right],
$$

respectively. It is obvious that both the branching ratio of $l_{\alpha}^{-} \rightarrow l_{\beta}^{-}+\gamma$ and the muon $g-2$ are enhanced by the masses of up-type quarks, especially that of top quark, and if there is no mixing between $S_{1}$ and $\widetilde{R}_{2}^{-\frac{1}{3}}$ (i.e., $\theta=0$ ) or the masses of the physical $S_{1}$ and $\widetilde{R}_{2}^{-\frac{1}{3}}$ are exactly degenerate (i.e., $M_{1}=M_{2}$ ), the results in eqs. (2.25) and (2.26) will recover the results caused by $S_{1}$ all alone [102].

\section{$3 \quad$ Neutrino masses and lepton flavor mixing}

As can be seen in section 2, the Yukawa coupling matrices $\lambda$ and $\lambda^{\mathrm{L}}$ contribute to the neutrino mass matrix, and both the radiative decays of charged leptons and the muon $g-2$ involve the Yukawa coupling matrices $\lambda^{\mathrm{L}}$ and $\lambda^{\mathrm{R}}$. In order to explain the neutrino oscillation observables and the anomaly of muon $g-2$, meanwhile satisfy the constraints from the radiative decays of charged leptons, we assume that the Yukawa coupling matrices have the following textures:

$$
\lambda=\left(\begin{array}{ccc}
0 & 0 & 0 \\
b(1+\varepsilon) & 0 & c(1+\varepsilon) \\
\frac{m_{\mathrm{s}}}{m_{\mathrm{b}}} b^{*} & \frac{m_{\mathrm{s}}}{m_{\mathrm{b}}} c^{*} & d
\end{array}\right), \quad \lambda^{\mathrm{L}}=a\left(\begin{array}{lll}
0 & 0 & 0 \\
0 & 0 & 1 \\
0 & 1 & 0
\end{array}\right), \quad \lambda^{\mathrm{R}}=a\left(\begin{array}{ccc}
0 & 0 & 0 \\
0 & x & 0 \\
0 & 1 & 0
\end{array}\right)
$$

with $\varepsilon$ being a small and real quantity, as well as $a$ and $d$ being real. The textures of $\lambda$ and $\lambda^{\mathrm{L}}$ are motivated by the fact that the lepton flavor mixing approximately has the $\mu-\tau$ symmetry $[1,124]$. The $(3,2)$ elements of $\lambda^{\mathrm{L}}$ and $\lambda^{\mathrm{R}}$ are responsible for explaining the muon $g-2$, while the $(2,2)$ element of $\lambda^{\mathrm{R}}$ is used to suppress the constraint from the LFV decay of tauon (i.e., $\tau \rightarrow \mu+\gamma$ ), which has been largely enhanced.

By means of eqs. (2.10) and (3.1), one can obtain the neutrino mass matrix, that is

$$
M_{\nu}=\frac{3 \sin 2 \theta}{32 \pi^{2}} \ln \left(\frac{M_{2}^{2}}{M_{1}^{2}}\right) a m_{\mathrm{b}}\left(\begin{array}{ccc}
0 & \frac{m_{\mathrm{s}}}{m_{\mathrm{b}}} b & \frac{m_{\mathrm{s}}}{m_{\mathrm{b}}} b^{*}(1+\varepsilon) \\
\frac{m_{\mathrm{s}}}{m_{\mathrm{b}}} & 2 \frac{m_{\mathrm{s}}}{m_{\mathrm{b}}} c & d \\
\frac{m_{\mathrm{s}}}{m_{\mathrm{b}}} b^{*}(1+\varepsilon) & d & 2 \frac{m_{\mathrm{s}}}{m_{\mathrm{b}}} c^{*}(1+\varepsilon)
\end{array}\right),
$$

whose $(1,1)$ element vanishes (i.e., $\left.\left(M_{\nu}\right)_{e e}=0\right)$ thanks to the vanishing elements in the first line and the first column of $\lambda^{\mathrm{L}}$ given in eq. (3.1). It is worth emphasizing that this novel feature is only in favor of the normal neutrino mass ordering. In the basis where the charged-lepton mass matrix is diagonal, $M_{\nu}$ can be diagonalized by the Pontecorvo-Maki-Nakagawa-Sakata (PMNS) matrix $U$ [133-135], namely, $U^{\dagger} M_{\nu} U^{*}=D_{\nu}$ with $D_{\nu} \equiv \operatorname{Diag}\left\{m_{1}, m_{2}, m_{3}\right\}$. In the standard parametrization [2], the PMNS matrix $U$ 
can be decomposed as

$$
U=P_{l}\left(\begin{array}{ccc}
c_{12} c_{13} & s_{12} c_{13} & s_{13} e^{-\mathrm{i} \delta_{\mathrm{CP}}} \\
-s_{12} c_{23}-c_{12} s_{13} s_{23} e^{\mathrm{i} \delta_{\mathrm{CP}}} & c_{12} c_{23}-s_{12} s_{13} s_{23} e^{\mathrm{i} \delta_{\mathrm{CP}}} & c_{13} s_{23} \\
s_{12} s_{23}-c_{12} s_{13} c_{23} e^{\mathrm{i} \delta_{\mathrm{CP}}} & -c_{12} s_{23}-s_{12} s_{13} c_{23} e^{\mathrm{i} \delta_{\mathrm{CP}}} & c_{13} c_{23}
\end{array}\right) P_{\nu}
$$

where $c_{i j}=\cos \theta_{i j}$ and $s_{i j}=\sin \theta_{i j}($ for $i j=12,13,23), P_{l}=\operatorname{Diag}\left\{e^{\mathrm{i} \phi_{e}}, e^{\mathrm{i} \phi_{\mu}}, e^{\mathrm{i} \phi_{\tau}}\right\}$ contains three unphysical phases, and $P_{\nu}=\operatorname{Diag}\left\{e^{\mathrm{i} \rho}, e^{\mathrm{i} \sigma}, 1\right\}$ is the Majorana phase matrix.

If $\varepsilon=0$, the neutrino mass matrix $M_{\nu}$ in eq. (3.2) will preserve the $\mu-\tau$ reflection symmetry $[123,124]$. For simplicity, we rewrite $M_{\nu}$ into

$$
M_{\nu}^{\mu-\tau}=\left(\begin{array}{ccc}
0 & B & B^{*} \\
B & C & D \\
B^{*} & D & C^{*}
\end{array}\right)
$$

in the limit of the $\mu-\tau$ reflection symmetry, where $B=3 \sin 2 \theta /\left(32 \pi^{2}\right) \ln \left(M_{2}^{2} / M_{1}^{2}\right) m_{\mathrm{s}} a b$, $C=6 \sin 2 \theta /\left(32 \pi^{2}\right) \ln \left(M_{2}^{2} / M_{1}^{2}\right) m_{\mathrm{s}} a c$ and $D=3 \sin 2 \theta /\left(32 \pi^{2}\right) \ln \left(M_{2}^{2} / M_{1}^{2}\right) m_{\mathrm{b}} a d$. It is wellknown that the $\mu-\tau$ reflection structure leads us to $[136,137]$

$$
\theta_{23}=\frac{\pi}{4}, \quad \delta_{\mathrm{CP}}= \pm \frac{\pi}{2}, \quad \rho, \sigma=0 \text { or } \frac{\pi}{2}, \quad \phi_{e}=0 \text { or } \frac{\pi}{2}, \quad \phi_{\mu}+\phi_{\tau}=2 \phi_{e} \pm \pi .
$$

In addition, we can obtain simple relationships between the other two mixing angles together with three neutrino masses and the elements of $M_{\nu}^{\mu-\tau}$, namely [137-140]

$$
\left\{\begin{array} { r l } 
{ \operatorname { t a n } \theta _ { 1 3 } } & { = \mathrm { i } e ^ { \mathrm { i } \delta } \frac { \operatorname { I m } ( C ^ { \prime } ) } { \sqrt { 2 } \operatorname { R e } ( B ^ { \prime } ) } } \\
{ \operatorname { t a n } 2 \theta _ { 1 2 } } & { = \frac { \sqrt { 2 } \operatorname { R e } ( B ^ { \prime } ) \operatorname { I m } ( C ^ { \prime } ) } { c _ { 1 3 } [ \operatorname { R e } ( C ^ { \prime } ) \operatorname { I m } ( C ^ { \prime } ) - \operatorname { R e } ( B ^ { \prime } ) \operatorname { I m } ( B ^ { \prime } ) ] } } \\
{ m _ { 1 } ^ { \rho } } & { = - D ^ { \prime } + \frac { \operatorname { R e } ( B ^ { \prime } ) \operatorname { I m } ( B ^ { \prime } ) } { \operatorname { I m } ( C ^ { \prime } ) } - \frac { \sqrt { 2 } \operatorname { R e } ( B ) } { c _ { 1 3 } \operatorname { s i n } 2 \theta _ { 1 2 } } } \\
{ m _ { 2 } ^ { \sigma } } & { = - D ^ { \prime } + \frac { \operatorname { R e } ( B ^ { \prime } ) \operatorname { I m } ( B ^ { \prime } ) } { \operatorname { I m } ( C ^ { \prime } ) } + \frac { \sqrt { 2 } \operatorname { R e } ( B ) } { c _ { 1 3 } \operatorname { s i n } 2 \theta _ { 1 2 } } } \\
{ m _ { 3 } } & { = \frac { 2 \operatorname { R e } ( B ^ { \prime } ) \operatorname { I m } ( B ^ { \prime } ) } { \operatorname { I m } ( C ^ { \prime } ) } }
\end{array} \quad \left\{\begin{array}{rl}
\operatorname{Re}\left(B^{\prime}\right)=\frac{\left(m_{2}^{\sigma}-m_{1}^{\rho}\right) c_{13} \sin 2 \theta_{12}}{2 \sqrt{2}} \\
\operatorname{Im}\left(B^{\prime}\right)=\mathrm{i} e^{\mathrm{i} \delta \frac{m_{3} \tan \theta_{13}}{\sqrt{2}}} \\
\operatorname{Re}\left(C^{\prime}\right)=\frac{\left(m_{2}^{\sigma}-m_{1}^{\rho}\right) \cos 2 \theta_{12}+m_{3}}{2} \\
\operatorname{Im}\left(C^{\prime}\right)=\mathrm{i} e^{\mathrm{i} \delta \frac{\left(m_{2}^{\sigma}-m_{1}^{\rho}\right) s_{13} \sin 2 \theta_{12}}{2}} \\
D^{\prime}=\frac{m_{3}-m_{2}^{\sigma}-m_{1}^{\rho}}{2}
\end{array}\right.\right.
$$

as well as

$$
\begin{array}{r}
m_{1}^{\rho} \cos ^{2} \theta_{12}+m_{2}^{\sigma} \sin ^{2} \theta_{12}-m_{3} \tan ^{2} \theta_{13}=0, \\
\operatorname{Re}\left(B^{\prime}\right) \operatorname{Im}\left(C^{\prime}\right)\left[\operatorname{Re}\left(C^{\prime}\right)+D^{\prime}\right]-\operatorname{Im}\left(B^{\prime}\right)\left[2\left(\operatorname{Re}\left(B^{\prime}\right)\right)^{2}-\left(\operatorname{Im}\left(C^{\prime}\right)\right)^{2}\right]=0,
\end{array}
$$

where $m_{1}^{\rho}=m_{1} \exp (2 \mathrm{i} \rho), m_{2}^{\sigma}=m_{2} \exp (2 \mathrm{i} \sigma), B^{\prime}=B \exp \left[-\mathrm{i}\left(\phi_{e}+\phi_{\mu}\right)\right], C^{\prime}=C \exp \left(-2 \mathrm{i} \phi_{\mu}\right)$ and $D^{\prime}=D \exp \left[-\mathrm{i}\left(\phi_{\mu}+\phi_{\tau}\right)\right]$. It is interesting that there exists a relation between the mixing parameters and neutrino masses or between the elements of $M_{\nu}^{\mu-\tau}$ and unphysical phases, resulting from the absence of $(0,0)$ element of $M_{\nu}^{\mu-\tau}$, as shown in eq. (3.7). Thus the mixing angles and neutrino masses are not independent. If the experimental values of $\Delta m_{21}^{2}, \Delta m_{31}^{2}$ or $\Delta m_{32}^{2}, \theta_{12}$ and $\theta_{13}$ are taken into account, one will find that only the normal neutrino mass ordering (i.e., $m_{1}<m_{2}<m_{3}$ ) can satisfy the first equation in eq. (3.7) with 
two possible values of $m_{1}$ corresponding to $(\rho, \sigma)=(\pi / 2,0)$ and $(0, \pi / 2)$, respectively, and the inverted neutrino mass ordering (i.e., $m_{3}<m_{1}<m_{2}$ ) is ruled out. By the way, the second equation in eq. (3.7) can be used to determinate the unphysical phase $\phi_{\mu}$ or $\phi_{\tau}$, only one of which is independent.

Now we consider $\varepsilon$ as a small quantity to break the $\mu-\tau$ reflection symmetry, then the neutrino masses and mixing parameters will gain corresponding corrections, namely, $\Delta m_{i}=m_{i}^{\prime}-m_{i}($ for $i=1,2,3), \Delta \theta_{i j}=\theta_{i j}^{\prime}-\theta_{i j}($ for $i j=12,13,23), \Delta \delta=\delta_{\mathrm{CP}}^{\prime}-\delta_{\mathrm{CP}}$, $\Delta \rho=\rho^{\prime}-\rho$ and $\Delta \sigma=\sigma^{\prime}-\sigma$ where the parameters with superscripts " $"$ "are the neutrino masses and mixing parameters for $\varepsilon \neq 0$. The PMNS matrix $U^{\prime}$ to diagonalize the mass matrix with $\varepsilon \neq 0$ in eq. (3.2) consists of those new mixing parameters and three new unphysical phases $\phi_{\alpha}^{\prime}$ (for $\left.\alpha=e, \mu, \tau\right)$. Following the method described in ref. [141], one can obtain

$$
\begin{aligned}
& \Delta m_{i} \simeq \operatorname{Re}\left(U^{\dagger} \Delta M_{\nu} U^{*}\right)_{i i}, \\
& \operatorname{Im}\left(\Delta U^{\dagger} U\right)_{i i} \simeq-\frac{\operatorname{Im}\left(U^{\dagger} \Delta M_{\nu} U^{*}\right)_{i i}}{2 m_{i}}, \\
& \operatorname{Re}\left(\Delta U^{\dagger} U\right)_{j k} \simeq \frac{\operatorname{Re}\left(U^{\dagger} \Delta M_{\nu} U^{*}\right)_{j k}}{m_{j}-m_{k}}, \\
& \operatorname{Im}\left(\Delta U^{\dagger} U\right)_{j k} \simeq-\frac{\operatorname{Im}\left(U^{\dagger} \Delta M_{\nu} U^{*}\right)_{j k}}{m_{j}+m_{k}},
\end{aligned}
$$

for $i=1,2,3$ and $j k=12,13,23$, where $\Delta M_{\mu} \equiv M_{\nu}-M_{\nu}^{\mu-\tau}$ and $\Delta U \equiv U^{\prime}-U$. Solving above linear equations leads us to

$$
\begin{aligned}
\Delta m_{1} \simeq & \frac{\eta}{2} \varepsilon\left\{\operatorname{Re}\left(C^{\prime}\right) s_{12}^{2}-\sqrt{2} \operatorname{Re}\left(B^{\prime}\right) \sin 2 \theta_{12} c_{13}+s_{13}\left[\eta_{\delta} \operatorname{Im}\left(C^{\prime}\right) \sin 2 \theta_{12}-\operatorname{Re}\left(C^{\prime}\right) c_{12}^{2} s_{13}\right.\right. \\
& \left.\left.-2 \sqrt{2} \eta_{\delta} \operatorname{Im}\left(B^{\prime}\right) c_{12}^{2} c_{13}\right]\right\} \\
\Delta m_{2} \simeq & -\frac{\eta}{2} \varepsilon\left\{\operatorname{Re}\left(C^{\prime}\right) c_{12}^{2}+\sqrt{2} \operatorname{Re}\left(B^{\prime}\right) \sin 2 \theta_{12} c_{13}-s_{13}\left[\eta_{\delta} \operatorname{Im}\left(C^{\prime}\right) \sin 2 \theta_{12}+\operatorname{Re}\left(C^{\prime}\right) s_{12}^{2} s_{13}\right.\right. \\
& \left.\left.+2 \sqrt{2} \eta_{\delta} \operatorname{Im}\left(B^{\prime}\right) s_{12}^{2} c_{13}\right]\right\} \\
\Delta m_{3} \simeq & \frac{1}{2} \varepsilon c_{13}\left[\operatorname{Re}\left(C^{\prime}\right) c_{13}-2 \sqrt{2} \eta_{\delta} \operatorname{Im}\left(B^{\prime}\right) s_{13}\right]
\end{aligned}
$$

and

$$
\begin{aligned}
\Delta \theta_{12} \simeq & \frac{\varepsilon}{2}\left\{\frac{1}{2} \sin 2 \theta_{12} s_{13}\left(\operatorname{Re}\left(C^{\prime}\right) s_{13}+\sqrt{2} \eta_{\delta} \operatorname{Im}\left(B^{\prime}\right) \cos 2 \theta_{13} \sec \theta_{13}\right)\left(\frac{1}{m_{3}-\eta m_{2}}-\frac{1}{m_{3}+\eta m_{1}}\right)\right. \\
& +s_{13}\left(\eta_{\delta} \operatorname{Im}\left(C^{\prime}\right)+\sqrt{2} \operatorname{Re}\left(B^{\prime}\right) \tan \theta_{13}\right)\left(\frac{s_{12}^{2}}{m_{3}+\eta m_{1}}+\frac{c_{12}^{2}}{m_{3}-\eta m_{2}}\right) \\
& +\left[\frac{1}{2} \sin 2 \theta_{12}\left(\operatorname{Re}\left(C^{\prime}\right)\left(1+s_{13}^{2}\right)+\sqrt{2} \eta_{\delta} \operatorname{Im}\left(B^{\prime}\right) \sin 2 \theta_{13}\right)+\cos 2 \theta_{12}\right. \\
& \left.\left.\times\left(\eta_{\delta} \operatorname{Im}\left(C^{\prime}\right) s_{13}-\sqrt{2} \operatorname{Re}\left(B^{\prime}\right) c_{13}\right)\right] \frac{\eta}{m_{1}+m_{2}}\right\},
\end{aligned}
$$




$$
\begin{aligned}
& \Delta \theta_{13} \simeq \frac{\varepsilon}{4}\left[\sin 2 \theta_{12}\left(\sqrt{2} \operatorname{Re}\left(B^{\prime}\right) s_{13}+\eta_{\delta} \operatorname{Im}\left(C^{\prime}\right) c_{13}\right)\left(\frac{1}{m_{3}+\eta m_{1}}-\frac{1}{m_{3}-\eta m_{2}}\right)\right. \\
& \left.-\left(\operatorname{Re}\left(C^{\prime}\right) \sin 2 \theta_{13}+2 \sqrt{2} \eta_{\delta} \operatorname{Im}\left(B^{\prime}\right) \cos 2 \theta_{13}\right)\left(\frac{c_{12}^{2}}{m_{3}+\eta m_{1}}+\frac{s_{12}^{2}}{m_{3}-\eta m_{2}}\right)\right], \\
& \Delta \theta_{23} \simeq \frac{\varepsilon}{2}\left[\frac{1}{2} \sin 2 \theta_{12}\left(\sqrt{2} \operatorname{Re}\left(B^{\prime}\right) \cos 2 \theta_{13} \sec \theta_{13}-\eta_{\delta} \operatorname{Im}\left(C^{\prime}\right) s_{13}\right)\left(\frac{1}{m_{3}-\eta m_{1}}-\frac{1}{m_{3}+\eta m_{2}}\right)\right. \\
& \left.+\left(\sqrt{2} \eta_{\delta} \operatorname{Im}\left(B^{\prime}\right) \tan \theta_{13}-\operatorname{Re}\left(C^{\prime}\right)\right)\left(\frac{c_{12}^{2}}{m_{3}+\eta m_{2}}+\frac{s_{12}^{2}}{m_{3}-\eta m_{1}}\right)\right], \\
& \Delta \delta_{\mathrm{CP}} \simeq-\frac{\varepsilon}{2}\left\{\left(2 \sqrt{2} \eta_{\delta} \operatorname{Re}\left(B^{\prime}\right) \cot 2 \theta_{13}-\operatorname{Im}\left(C^{\prime}\right)\right)\left(\frac{\cos 2 \theta_{12}-c_{13}^{2} c_{12}^{2}}{m_{3}+\eta m_{2}}-\frac{\cos 2 \theta_{12}+c_{13}^{2} s_{12}^{2}}{m_{3}-\eta m_{1}}\right)\right. \\
& +\left(\eta_{\delta} \operatorname{Re}\left(C^{\prime}\right) \csc \theta_{13}-\sqrt{2} \operatorname{Im}\left(B^{\prime}\right) \sec \theta_{13}\right)\left[\frac{\cot \theta_{12}\left(\cos 2 \theta_{12}-c_{13}^{2} c_{12}^{2}\right)}{m_{3}+\eta m_{2}}\right. \\
& \left.+\frac{\tan \theta_{12}\left(\cos 2 \theta_{12}+c_{13}^{2} s_{12}^{2}\right)}{m_{3}-\eta m_{1}}\right]-\left[2 \cot 2 \theta_{12}\left(\sqrt{2} \operatorname{Im}\left(B^{\prime}\right) c_{13}+\eta_{\delta} \operatorname{Re}\left(C^{\prime}\right) s_{13}\right)\right. \\
& \left.\left.+\sqrt{2} \eta_{\delta} \operatorname{Re}\left(B^{\prime}\right) \sin 2 \theta_{13}-\operatorname{Im}\left(C^{\prime}\right)\left(1+s_{13}^{2}\right)\right] \frac{\eta}{m_{2}-m_{1}}\right\}, \\
& \Delta \rho \simeq \frac{\varepsilon}{4}\left[2 s_{13}\left(\eta_{\delta} \operatorname{Re}\left(C^{\prime}\right)-\sqrt{2} \operatorname{Im}\left(B^{\prime}\right) \tan \theta_{13}\right)\left(\frac{\cos 2 \theta_{12} \cot \theta_{12}}{m_{3}+\eta m_{2}}+\frac{\sin 2 \theta_{12}}{m_{3}-\eta m_{1}}\right)\right. \\
& -2\left(\sqrt{2} \eta_{\delta} \operatorname{Re}\left(B^{\prime}\right) \cos 2 \theta_{13} \tan \theta_{13}-\operatorname{Im}\left(C^{\prime}\right) s_{13}^{2}\right)\left(\frac{2 c_{12}^{2}}{m_{3}-\eta m_{1}}-\frac{\cos 2 \theta_{12}}{m_{3}+\eta m_{2}}\right) \\
& -\eta\left(\sqrt{2} \operatorname{Im}\left(B^{\prime}\right) c_{13}+\eta_{\delta} \operatorname{Re}\left(C^{\prime}\right) s_{13}\right)\left(\frac{2 \cot \theta_{12} \cos 2 \theta_{12}}{m_{2}-m_{1}}-\frac{\sin 2 \theta_{12}}{m_{1}}\right) \\
& -\left(\operatorname{Im}\left(C^{\prime}\right) c_{13}^{2}+\sqrt{2} \eta_{\delta} \operatorname{Re}\left(B^{\prime}\right) \sin 2 \theta_{13}\right)\left(\eta \frac{c_{12}^{2}}{m_{1}}+\eta \frac{2 c_{12}^{2}}{m_{2}-m_{1}}-\frac{1}{m_{3}}\right) \\
& \left.+\eta \operatorname{Im}\left(C^{\prime}\right)\left(\frac{4 c_{12}^{2}}{m_{2}-m_{1}}+\frac{\cos 2 \theta_{12}}{m_{1}}\right)\right], \\
& \Delta \sigma \simeq \frac{\varepsilon}{4}\left[2 s_{13}\left(\sqrt{2} \operatorname{Im}\left(B^{\prime}\right) \tan \theta_{13}-\eta_{\delta} \operatorname{Re}\left(C^{\prime}\right)\right)\left(\frac{\sin 2 \theta_{12}}{m_{3}+\eta m_{2}}-\frac{\cos 2 \theta_{12} \tan \theta_{12}}{m_{3}-\eta m_{1}}\right)\right. \\
& -2\left(\sqrt{2} \eta_{\delta} \operatorname{Re}\left(B^{\prime}\right) \cos 2 \theta_{13} \tan \theta_{13}-\operatorname{Im}\left(C^{\prime}\right) s_{13}^{2}\right)\left(\frac{\cos 2 \theta_{12}}{m_{3}-\eta m_{1}}+\frac{2 s_{12}^{2}}{m_{3}+\eta m_{2}}\right) \\
& +\eta\left(\sqrt{2} \operatorname{Im}\left(B^{\prime}\right) c_{13}+\eta_{\delta} \operatorname{Re}\left(C^{\prime}\right) s_{13}\right)\left(\frac{\sin 2 \theta_{12}}{m_{2}}-\frac{2 \tan \theta_{12} \cos 2 \theta_{12}}{m_{2}-m_{1}}\right) \\
& +\left(\operatorname{Im}\left(C^{\prime}\right) c_{13}^{2}+\sqrt{2} \eta_{\delta} \operatorname{Re}\left(B^{\prime}\right) \sin 2 \theta_{13}\right)\left(\frac{1}{m_{3}}+\eta \frac{s_{12}^{2}}{m_{2}}-\eta \frac{2 s_{12}^{2}}{m_{2}-m_{1}}\right) \\
& \left.+\eta \operatorname{Im}\left(C^{\prime}\right)\left(\frac{\cos 2 \theta_{12}}{m_{2}}+\frac{4 s_{12}^{2}}{m_{2}-m_{1}}\right)\right],
\end{aligned}
$$

where $\eta_{\delta}= \pm 1$ for $\delta_{\mathrm{CP}}= \pm \pi / 2$ and $\eta= \pm 1$ for $(\rho, \sigma)=(0, \pi / 2),(\pi / 2,0)$. It is worth pointing out that with the help of eqs. (3.6) and (3.7), one can achieve all the deviations 
given in eqs. (3.9) and (3.10) as functions either only of the neutrino masses and mixing angles or only of the elements of $M_{\nu}$. Here we focus on the deviations of $\theta_{23}$ and $\delta_{\mathrm{CP}}$, approximately given by

$$
\begin{aligned}
\Delta \theta_{23} & \simeq-\frac{\varepsilon}{4}, \\
\Delta \delta_{\mathrm{CP}} & \simeq \frac{\eta_{\delta} \varepsilon\left[1-r_{12}-\left(1+r_{12}\right) \cos 2 \theta_{12}\right] \cot 2 \theta_{12}}{4\left(1-r_{12}\right) \theta_{13}},
\end{aligned}
$$

at the leading order with $r_{12}=m_{1} / m_{2}$, where eqs. (3.6) and (3.7) have been taken into account. Note that the deviation of $\theta_{23}$ is simply proportional to $\varepsilon$ and that of $\delta_{\mathrm{CP}}$ is enhanced by the smallness of $\theta_{13}$.

\section{Numerical calculations}

There are thirteen relevant real parameters $\left\{M_{i}, \theta, a, \operatorname{Re}(b), \operatorname{Im}(b), \operatorname{Re}(c), \operatorname{Im}(c), d, \operatorname{Re}(x)\right.$, $\operatorname{Im}(x), \varepsilon\}$ (for $i=1,2,3$ ) involved in our model with the chosen Yukawa coupling matrices in eq. (3.1) to confront with the muon $g-2$, constraints from LFV decays of charged leptons, neutrino masses and neutrino mixing parameters. For simplicity, we assume that the mixing between $S_{1}$ and $\widetilde{R}_{2}^{-\frac{1}{3} *}$ is maximal, namely $\theta=\pi / 4$, and masses of the physical LQs satisfy $M_{1}=M_{2} /(1+r)=M_{3}=2 \mathrm{TeV}$ with $r$ defined as $r \equiv\left(M_{2}-M_{1}\right) / M_{1}$. We take $r$ as a small quantity, which means that masses of the physical $S_{1}$ and $\widetilde{R}_{2}^{-\frac{1}{3} *}$ are nearly degenerate and is required to give the correct scale of neutrino masses. As we will see, the parameter $x$ can be taken to be a complex constant and used to suppress the constraint from $\tau^{-} \rightarrow \mu^{-}+\gamma$. Thus, only eight parameters $\{r, a, \operatorname{Re}(b), \operatorname{Im}(b), \operatorname{Re}(c), \operatorname{Im}(c), d, \varepsilon\}$ are left.

The masses of charged fermions used in the numerical calculations are their central values at $M_{Z}[142]:^{5}$

$$
\begin{array}{lll}
m_{\mathrm{u}}=1.23 \mathrm{MeV}, & m_{\mathrm{c}}=0.620 \mathrm{GeV}, & m_{\mathrm{t}}=168.26 \mathrm{GeV}, \\
m_{\mathrm{d}}=2.67 \mathrm{MeV}, & m_{\mathrm{s}}=53.16 \mathrm{MeV}, & m_{\mathrm{b}}=2.839 \mathrm{GeV}, \\
m_{e}=0.48307 \mathrm{MeV}, & m_{\mu}=0.101766 \mathrm{MeV}, & m_{\tau}=1.72856 \mathrm{GeV},
\end{array}
$$

and the quark mixing parameters under the standard parametrization also take their central values [2]:

$$
\sin \theta_{12}^{\mathrm{q}}=0.22650, \quad \sin \theta_{13}^{\mathrm{q}}=0.00361, \quad \sin \theta_{23}^{\mathrm{q}}=0.04053, \quad \delta^{\mathrm{q}}=1.196 .
$$

The difference between the SM value and the combined experimental average value of the muon anomalous magnetic moment with $1 \sigma$ error is [21]

$$
\Delta a_{\mu}=(251 \pm 59) \times 10^{-11},
$$

\footnotetext{
${ }^{5}$ In principle, one should take the pole masses of charged fermions for precision calculations. Here for illustration, we naively make use of their running masses at $M_{Z}$ to show that our model can well accommodate the low-energy observables we are concerned about. The differences between pole masses and running masses at $M_{Z}$ which come from high-order corrections in the perturbative calculations have very limited effects on the numerical analyses.
} 
and the current experimental upper bounds on the branching ratios of radiative $l_{\alpha}^{-} \rightarrow l_{\beta}^{-}+\gamma$ decays are

$$
\begin{aligned}
& \mathcal{B}\left(\mu^{-} \rightarrow e^{-}+\gamma\right)<4.2 \times 10^{-13} \\
& \mathcal{B}\left(\tau^{-} \rightarrow e^{-}+\gamma\right)<3.3 \times 10^{-8} \\
& \mathcal{B}\left(\tau^{-} \rightarrow \mu^{-}+\gamma\right)<4.4 \times 10^{-8}
\end{aligned}
$$

at the $90 \%$ confidence level [2]. The best-fit values with $1 \sigma$ errors of two neutrino masssquared differences and neutrino mixing parameters in the normal neutrino mass ordering are $[143]$

$$
\begin{aligned}
\sin ^{2} \theta_{12} & =0.304_{-0.012}^{+0.013}, & \sin ^{2} \theta_{13} & =0.02221_{-0.00062}^{+0.00068}, \\
\sin ^{2} \theta_{23} & =0.570_{-0.024}^{+0.018}, & \delta_{\mathrm{CP}} & =\left(195_{-25}^{+51}\right)^{\circ}, \\
\Delta m_{21}^{2} & =\left(7.42_{-0.20}^{+0.21}\right) \times 10^{-5} \mathrm{eV}^{2}, & \Delta m_{31}^{2} & =\left(2.514_{-0.027}^{+0.028}\right) \times 10^{-3} \mathrm{eV}^{2} .
\end{aligned}
$$

To find out the allowed regions of model parameters, with which the predictions for low-energy observables can be compatible with the experimental data shown in eqs. (4.3)(4.5), we define the Gaussian- $\chi^{2}$ function as

$$
\chi^{2}=\sum_{j}\left(\frac{q_{j}\left(p_{i}\right)-q_{j}^{\mathrm{bf}}}{\sigma_{j}}\right)^{2},
$$

where $p=\{r, a, \operatorname{Re}(b), \operatorname{Im}(b), \operatorname{Re}(c), \operatorname{Im}(c), d, \varepsilon\}$ are model parameters, $q=\left\{\Delta m_{21}^{2}, \Delta m_{31}^{2}\right.$, $\left.\sin ^{2} \theta_{12}, \sin ^{2} \theta_{13}, \sin ^{2} \theta_{23}, \Delta a_{\mu}\right\}$ are low-energy observables, $q_{j}\left(p_{i}\right)$ represent the model predictions for these observables, $q_{j}^{\text {bf }}$ stand for the best-fit values of these observables given in eqs. (4.3) and (4.5), and $\sigma_{j}$ are the symmetrized $1 \sigma$ errors. Here we do not include the information of $\delta_{\mathrm{CP}}$ in the $\chi^{2}$-function as a result of the weak constraint on it from the current global-fit results [143]. Then minimizing the $\chi^{2}$-function defined in eq. (4.6), we can obtain the best-fit values of the model parameters $p_{i}$, and we shall directly use $N \sigma=\sqrt{\chi^{2}}$ to derive the allowed parameter ranges at $N$ standard deviations.

Before carrying out the numerical calculations, we first discuss some features of the muon $g-2$, the radiative decays of charged leptons and the flavor structure of neutrinos with the chosen Yukawa coupling matrices in eq. (3.1), which will give guidance on the numerical analyses.

- Given the Yukawa coupling matrices in eq. (3.1), only the branching ratio of $\tau^{-} \rightarrow$ $\mu^{-}+\gamma$ is largely enhanced and has the possibility to exceed the experimental constraint given in eq. (4.4). To avoid this excess, we take the $(2,2)$ element of $\lambda^{R}$ to be

$$
x=-\frac{m_{\mathrm{t}} V_{\mathrm{cb}}^{*} \mathcal{F}\left(\frac{m_{\mathrm{t}}^{2}}{M_{1}^{2}}\right)}{m_{\mathrm{c}} V_{\mathrm{cS}}^{*} \mathcal{F}\left(\frac{m_{\mathrm{c}}^{2}}{M_{1}^{2}}\right)} \simeq-2.59+8.2 \times 10^{-5} \mathrm{i},
$$


with the inputs shown in eqs. (4.1) and (4.2). Then by means of eq. (2.25), the branching ratio of $\tau^{-} \rightarrow \mu^{-}+\gamma$ is approximately given by

$$
\mathcal{B}\left(\tau^{-} \rightarrow \mu^{-}+\gamma\right) \simeq \frac{\alpha_{\mathrm{em}} m_{\tau}^{3} m_{\mathrm{t}}^{2}\left|V_{\mathrm{cb}}\right|^{2} a^{4} r^{2}}{16 \pi^{4} M_{1}^{4} \Gamma_{\tau}}\left(\frac{\ln \frac{m_{\mathrm{t}}^{2}}{m_{\mathrm{c}}^{2}}}{7+4 \ln \frac{m_{\mathrm{c}}^{2}}{M_{1}^{2}}}\right)^{2} \sim 1 \times 10^{-6} a^{4} r^{2},
$$

which can easily satisfy the experimental constraint with small $a$ and $r$.

- With the help of eqs. (2.26), (3.1) and (4.7), one may obtain

$$
\Delta a_{\mu} \simeq \frac{m_{\mu} m_{\mathrm{t}} a^{2}}{4 \pi^{2} M_{1}^{2}} \operatorname{Re}\left(V_{\mathrm{tb}}-\frac{V_{\mathrm{cb}} V_{\mathrm{ts}}}{V_{\mathrm{cs}}}\right)\left(\ln \frac{M_{1}^{2}}{m_{\mathrm{t}}^{2}}-\frac{7}{4}\right) \sim 251 \times 10^{-11}\left(\frac{a}{0.085}\right)^{2},
$$

which means that $a \sim 0.085$ can accommodate the experimental value of $\Delta a_{\mu}$ in eq. (4.3).

- The $(1,1)$ element of $M_{\nu}$ given in eq. (3.2) is vanishing, i.e., $\left(M_{\nu}\right)_{e e}=0$, resulting in the elimination of the inverted neutrino mass ordering in this model. This point also can be seen from eq. (3.7) when $\varepsilon$ is small. If we substitute the best-fit values of $\Delta m_{21}^{2}, \Delta m_{31}^{2}, \theta_{12}$ and $\theta_{13}$ into eq. (3.7) in the limit of $\mu-\tau$ reflection symmetry, we may obtain two solutions for $m_{1}: m_{1} \simeq 6.3 \mathrm{meV}$ for $(\rho, \sigma)=(0, \pi / 2)$ and $m_{1} \simeq 2.2$ $\mathrm{meV}$ for $(\rho, \sigma)=(\pi / 2,0)$. This infers that there will be two parameter ranges for $m_{1}$ around these two solutions when $M_{\nu}$ with a small $\varepsilon$ is considered.

- As can be seen in eq. (3.2), the mass scale of neutrinos is largely enhanced by $m_{\mathrm{b}}$. Since $a \sim 0.085$ is necessary to explain $\Delta a_{\mu}$, the masses $M_{1}$ and $M_{2}$ need to be highly but not exactly degenerate to get the right mass scale of neutrinos. Making use of the trace of $M_{\nu} M_{\nu}^{\dagger}$, we have

$$
r \sim \frac{16 \pi^{2}}{3 a m_{\mathrm{b}}} \sqrt{\frac{1}{2 d^{2}}\left(3 m_{1}^{2}+\Delta m_{21}^{2}+\Delta m_{31}^{2}\right)} \sim 8 \times 10^{-7}
$$

for $a \sim 0.085, d \sim \mathcal{O}(0.01)$ and $m_{1} \sim \mathcal{O}(0.01 \mathrm{eV})$.

Now carrying out the numerical calculations with all inputs, we find that the minimum of $\chi^{2}$-function is $\chi_{\min }^{2}=6.7 \times 10^{-6}$ and the corresponding best-fit values of the model parameters and the predicted low-energy observables are listed in the third column of table 1 . In addition, the $1 \sigma$ and $3 \sigma$ ranges of both the model parameters and the predicted low-energy observables are listed in the last two columns of table 1, respectively. To make results clearer, we plot correlations of some pairs of the predicted low-energy observables and also those between the predicted low-energy observables and model parameters in figures 3 and 4 . Some comments on the numerical results are in order.

- With the best-fit values of the model parameters listed in the third column of table 1, our model can successfully reproduce the experimental values of $\Delta m_{21}^{2}$, 


\begin{tabular}{|c|c|c|c|c|}
\hline & & Best-fit value & $1 \sigma$ range & $3 \sigma$ range \\
\hline \multirow{9}{*}{ 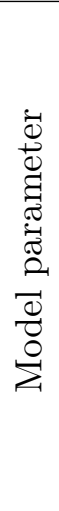 } & $a / 10^{-2}$ & 8.37 & $7.37 \rightarrow 9.24$ & $4.60 \rightarrow 10.9$ \\
\hline & $\mathrm{Bo}(h)$ & & $(-0.00245 \rightarrow 0.0000687)$ & $(-0.00260 \rightarrow 0.000613)$ \\
\hline & $\operatorname{Re}(D)$ & -0.000473 & $\cup(0.0961 \rightarrow 0.608)$ & $\cup(0.0748 \rightarrow 0.682)$ \\
\hline & $\operatorname{Im}(b)$ & 0.0246 & $-0.00410 \rightarrow 0.214$ & $-0.0192 \rightarrow 0.241$ \\
\hline & $\operatorname{Re}(c)$ & 0.000280 & $-0.0278 \rightarrow 0.196$ & $-0.0940 \rightarrow 0.237$ \\
\hline & $\operatorname{Im}(c)$ & 0.0367 & $0.0243 \rightarrow 1.28$ & $0.0146 \rightarrow 1.43$ \\
\hline & $d$ & 0.00147 & $0.000990 \rightarrow 0.0338$ & $0.000579 \rightarrow 0.0366$ \\
\hline & $r / 10^{-6}$ & 4.10 & $0.136 \rightarrow 5.89$ & $0.108 \rightarrow 10.1$ \\
\hline & $\varepsilon$ & -0.276 & $-0.342 \rightarrow-0.143$ & $-0.470 \rightarrow-0.0249$ \\
\hline \multirow{15}{*}{ 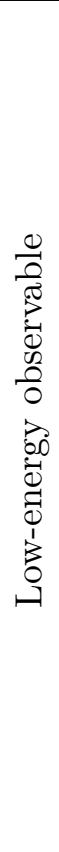 } & & 573 & $(2.12 \rightarrow 2.58)$ & $(1.70 \rightarrow 3.17)$ \\
\hline & $m_{1} / \mathrm{meV}$ & 5.73 & $\cup(5.28 \rightarrow 6.17)$ & $\cup(4.44 \rightarrow 7.24)$ \\
\hline & $\Delta m_{21}^{2} / 10^{-5} \mathrm{eV}^{2}$ & 7.42 & $7.22 \rightarrow 7.61$ & $6.81 \rightarrow 8.03$ \\
\hline & $\Delta m_{31}^{2} / 10^{-3} \mathrm{eV}^{2}$ & 2.51 & $2.49 \rightarrow 2.54$ & $2.43 \rightarrow 2.60$ \\
\hline & $\theta_{12} /^{\circ}$ & 33.46 & $32.71 \rightarrow 34.19$ & $31.13 \rightarrow 35.73$ \\
\hline & $\theta_{13} /{ }^{\circ}$ & 8.57 & $8.45 \rightarrow 8.69$ & $8.19 \rightarrow 8.94$ \\
\hline & $\theta_{23} /{ }^{\circ}$ & 49.03 & $47.89 \rightarrow 50.17$ & $45.44 \rightarrow 52.65$ \\
\hline & $\delta \quad 10$ & 24209 & $(236.52 \rightarrow 249.95)$ & $(224.14 \rightarrow 266.88)$ \\
\hline & & & $\cup(275.00 \rightarrow 279.01)$ & $\cup(270.82 \rightarrow 283.67)$ \\
\hline & $\rho / 0$ & 8.58 & $(6.47 \rightarrow 10.43)$ & $(1.03 \rightarrow 13.50)$ \\
\hline & $\rho /$ & & $\cup(94.02 \rightarrow 96.92)$ & $\cup(90.66 \rightarrow 99.91)$ \\
\hline & -10 & די 27 & $(0.29 \rightarrow 0.55)$ & $(0.05 \rightarrow 0.91)$ \\
\hline & $\sigma /$ & 92.21 & $\cup(91.73 \rightarrow 92.72)$ & $\cup(90.28 \rightarrow 93.55)$ \\
\hline & $\Delta a_{\mu} / 10^{-11}$ & 251.10 & $194.74 \rightarrow 305.97$ & $75.94 \rightarrow 426.33$ \\
\hline & $\mathcal{B}\left(\tau^{-} \rightarrow \mu^{-}+\gamma\right)$ & $1.17 \times 10^{-18}$ & $(0.71 \rightarrow 1.75) \times 10^{-18}$ & $(0.11 \rightarrow 3.39) \times 10^{-18}$ \\
\hline
\end{tabular}

Table 1. The best-fit values ( with $\chi_{\min }^{2}=6.7 \times 10^{-6}$ ) along with the $1 \sigma$ and $3 \sigma$ ranges of the model parameters and the predicted low-energy observables. The symbol " $\cup$ " stands for the union of two sets.

$\Delta m_{31}^{2}, \sin ^{2} \theta_{12}, \sin ^{2} \theta_{13}$ and $\sin ^{2} \theta_{23}$, and explain the muon anomalous magnetic moment $\Delta a_{\mu}$, as well as satisfy the constraints from the LFVs decays of charged leptons, with a quite small value of $\chi^{2}$-function, namely $\chi_{\min }^{2}=6.7 \times 10^{-6}$. Meanwhile, the lightest neutrino mass and three CP-violating phases are predicted to be $m_{1}=5.73$ $\mathrm{meV}, \delta_{\mathrm{CP}}=242.99^{\circ}, \rho=8.58^{\circ}$ and $\sigma=92.27^{\circ}$, respectively.

- As can seen from table 1 and also figure 3 , there are two disconnected ranges for $m_{1}, \delta_{\mathrm{CP}}, \rho$ and $\sigma$. The two disconnected ranges for $m_{1}, \rho$ and $\sigma$ can be easily understood in the limit of the $\mu-\tau$ reflection symmetry (i.e., $\varepsilon=0$ ), where two possible values of $m_{1}$ respectively associated with two specific combinational values of $\rho$ and $\sigma$ can be worked out by eq. (3.7), that is $m_{1}=2.2 \mathrm{meV}$ for $(\rho, \sigma)=(\pi / 2,0)$ 

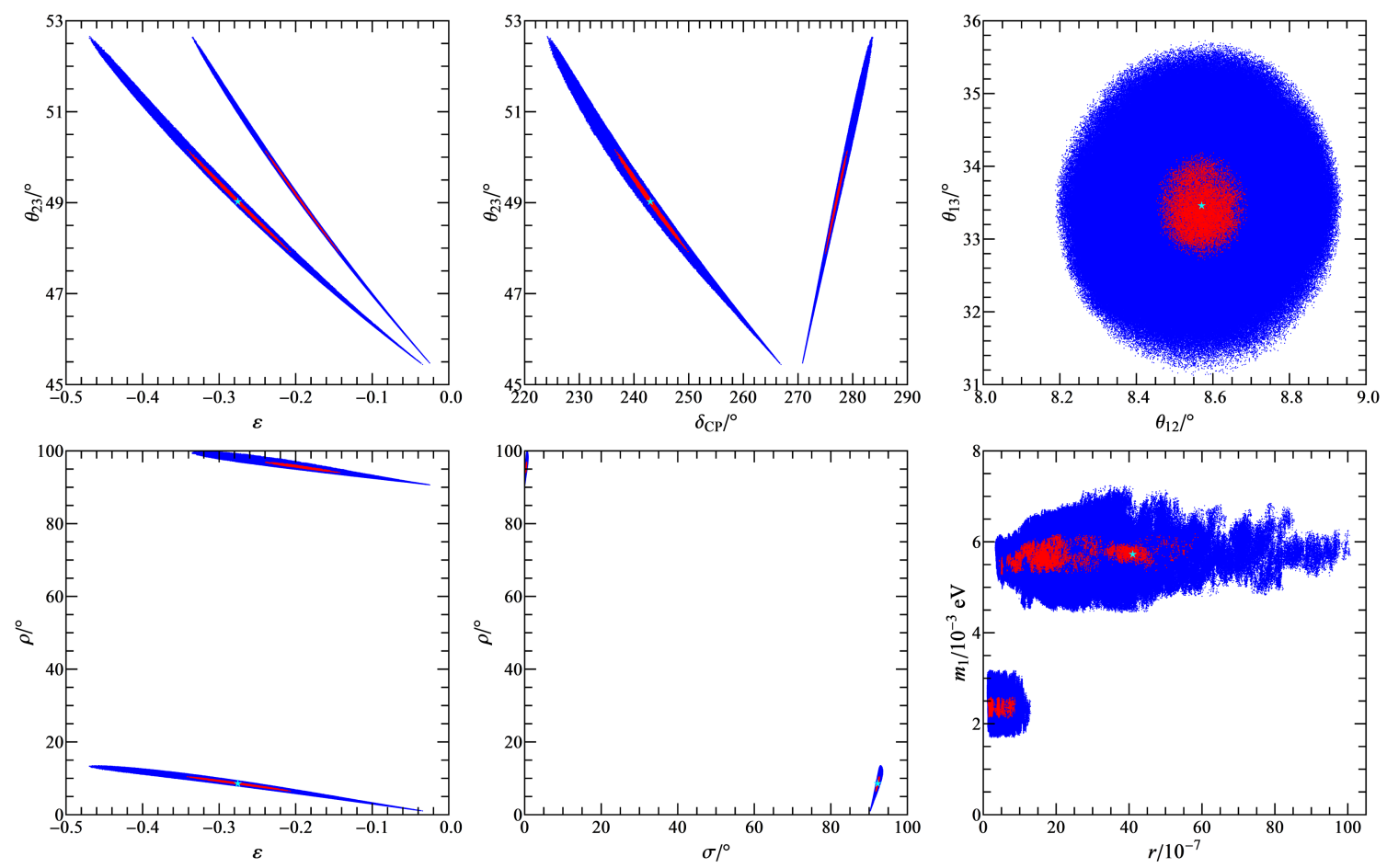

Figure 3. Correlations of the pairs $\left(\theta_{23}, \varepsilon\right),\left(\theta_{23}, \delta_{\mathrm{CP}}\right),\left(\theta_{13}, \theta_{12}\right),(\rho, \varepsilon),(\rho, \sigma)$ and $\left(m_{1}, r\right)$, where the cyan stars denote the best-fit values, and the red and blue regions correspond to parameter spaces at $1 \sigma$ and $3 \sigma$ levels, respectively.

and $m_{1}=6.3 \mathrm{meV}$ for $(\rho, \sigma)=(0, \pi / 2)$, as discussed above. Thus when the $\mu-\tau$ reflection symmetry is slightly broken, (in other words, $\varepsilon$ is small but not zero), the values of $m_{1}, \rho$ and $\sigma$ will be around these two possible solutions in the limit of $\mu-\tau$ reflection symmetry, which is exactly the case considered here. One may understand the two disconnected ranges for $\delta_{\mathrm{CP}}$ with the help of eq. (3.11). It can be easily checked that when the best-fit values of $\Delta m_{21}^{2}$ and $\theta_{12}$ in eq. (4.5) are taken into consideration, $1-r_{12}-\left(1+r_{12}\right) \cos 2 \theta_{12}$ is monotonically decreasing as a function of $m_{1}$ and $m_{1} \simeq 4 \mathrm{meV}$ is a zero of it. This leads to $\delta_{\mathrm{CP}}>270^{\circ}$ for $m_{1} \lesssim 4 \mathrm{meV}$ and $\delta_{\mathrm{CP}}<270^{\circ}$ for $m_{1} \gtrsim 4 \mathrm{meV}$ with initially $\delta_{\mathrm{CP}}=270^{\circ}$ in the limit of $\mu-\tau$ reflection symmetry, which is consistent with the obtained numerical results for $\delta_{\mathrm{CP}}$.

- The upper-left panel of figure 3 shows that the value of $\Delta \theta_{23}$ is approximately proportional to $-\varepsilon$, and $\theta_{23}$ is in the second octant for $\varepsilon<0$. These behaviors of $\theta_{23}$ coincide with the approximate analytical formula for the deviation of $\theta_{23}$ given in eq. (3.11), but in order to explain the two parameter spaces for $\theta_{23^{-}}{ }^{-}$, higher order terms involving $r_{12}$ in the analytical result for $\Delta \theta_{23}$ need to be restored. The deviation of $\delta_{\mathrm{CP}}$ shown in the upper-middle panel of figure 3 is much larger than other mixing parameters, which is actually enhanced by $1 / \theta_{13}$ as revealed in eq. (3.11). From the lower-right panel of figure 3, one can find that the maximal value $r$ can take for $m_{1} \sim 2.5 \mathrm{meV}$ is smaller than that for $m_{1} \sim 5.5 \mathrm{meV}$. This may be roughly understood with the help of eq. (4.10). 

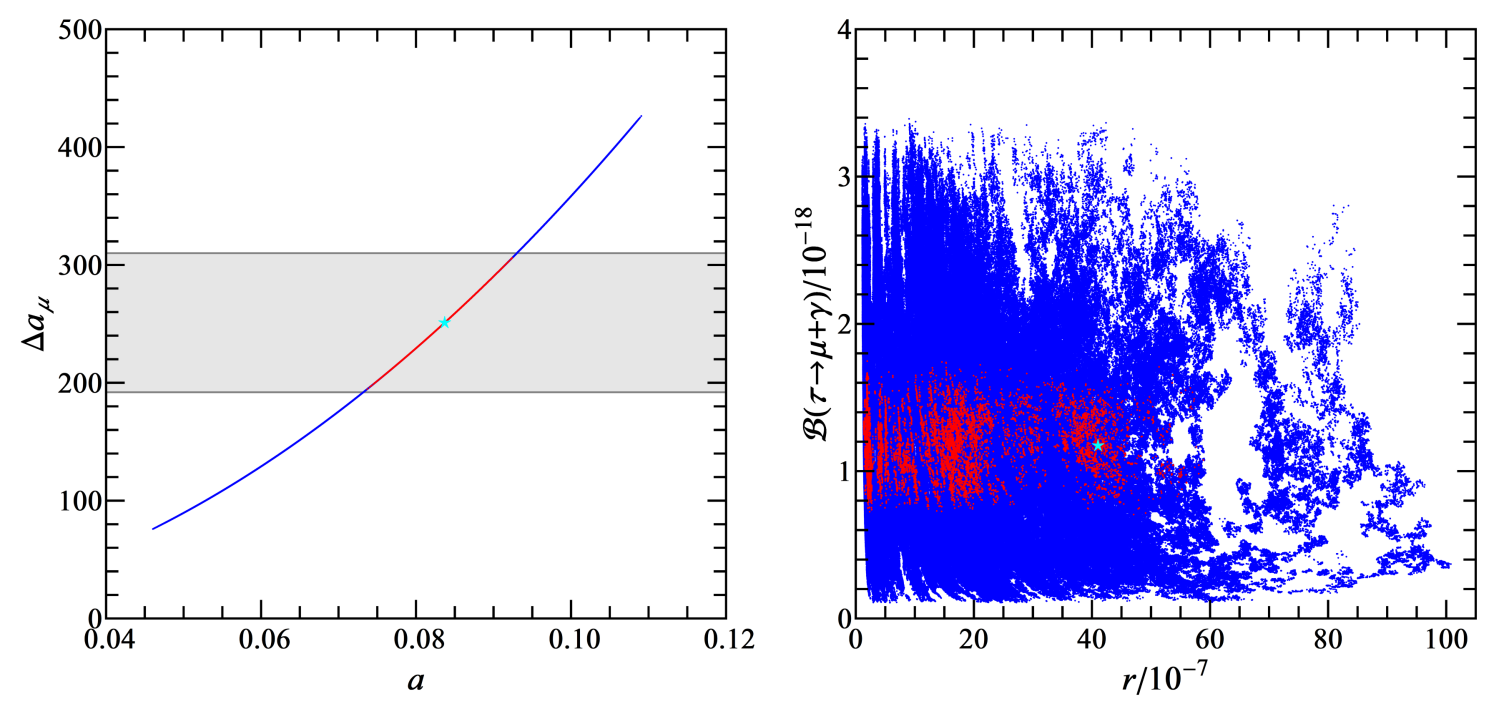

Figure 4. Correlation between $\Delta a_{\mu}$ and $a$, and that between $\mathcal{B}\left(\tau^{-} \rightarrow \mu^{-}+\gamma\right)$ and $r$, where the cyan stars denote the best-fit values, the gray region in the left panel is the experimental $1 \sigma$ range of $\Delta a_{\mu}$, and the red and blue regions correspond to parameter spaces at $1 \sigma$ and $3 \sigma$ levels, respectively.

- The approximate result for $\Delta a_{\mu}$ in eq. (4.9) where $\Delta a_{\mu}$ is a quadratic function of the model parameter $a$, perfectly describes the behavior of $\Delta a_{\mu}$ against $a$ shown in the left panel of figure 4. However, the approximate formula for the branching ratio $\mathcal{B}\left(\tau^{-} \rightarrow \mu^{-}+\gamma\right)$ given in eq. (4.8) predicts smaller values than those shown in the right panel of figure 4 . The reason is that for $r \sim 10^{-7}$, the masses of the physical $S_{1}$ and $\tilde{R}_{2}^{-\frac{1}{3}}$ are highly degenerate, leading to severe cancellation between the chirally enhanced contributions to $\mathcal{B}\left(\tau^{-} \rightarrow \mu^{-}+\gamma\right)$ from the physical $S_{1}$ and $\tilde{R}_{2}^{-\frac{1}{3}}$ with $x$ given in eq. (4.7), and then the contributions not enhanced (e.g., the second terms in the square brackets in eq. (2.13)) dominate the value of $\mathcal{B}\left(\tau^{-} \rightarrow \mu^{-}+\gamma\right)$ in the present case.

\section{Summary}

For the purpose of giving a combined explanation of the tiny neutrino masses, lepton flavor mixing and muon $g-2$, we have extended the SM with two TeV-scale scalar leptoquarks $S_{1}$ and $\widetilde{R}_{2}$. After constructing the complete Lagrangian with baryon number conservation, we calculate the neutrino mass matrix generated via one-loop quantum corrections, where the mixing between $S_{1}$ and $\widetilde{R}_{2}$ resulting from the lepton-number-violating LQ-Higgs interaction plays a greatly significant role. We also recalculate all the contributions to the radiative decays of charged leptons and the muon $g-2$ from all leptoquarks. The contributions from the physical $S_{1}$ and $\widetilde{R}_{2}^{-\frac{1}{3}}$ are quite similar and both largely enhanced by the up-type quark masses, which dominate the branching ratios of charged-lepton radiative decays and the size of the muon $g-2$. Given the textures of the leptoquark Yukawa coupling matrices shown in eq. (3.1), the neutrino mass matrix possesses an approximate $\mu-\tau$ reflection symmetry, and 
moreover its $(1,1)$ element vanishes, i.e., $\left(M_{\nu}\right)_{e e}=0$, which is only in favor of the normal neutrino mass ordering. Considering this novel texture of the neutrino mass matrix, we have analytically discussed its main features and the associated neutrino masses and lepton flavor mixing in detail. Finally, the numerical calculations show that our model can successfully explain the anomaly of the muon $g-2$ and accommodate the latest global-fit results of neutrino oscillation data, as well as satisfy the constraints from the radiative decays of charged leptons. More specifically, given the best-fit values of the model parameters with $\chi_{\min }^{2}=6.7 \times 10^{-6}$, the central value of $\Delta a_{\mu}$ and the best-fit values of $\Delta m_{21}^{2}, \Delta m_{31}^{2}$, $\theta_{12}, \theta_{13}$ and $\theta_{23}$ respectively given in eqs. (4.3) and (4.5) are reproduced pretty well, and additionally the lightest neutrino mass and three $\mathrm{CP}$-violating phases are predicted to be $m_{1}=5.73 \mathrm{meV}, \delta_{\mathrm{CP}}=242.99^{\circ}, \rho=8.58^{\circ}$ and $\sigma=92.27^{\circ}$. An interesting feature of our model is that the allowed ranges of $m_{1}, \rho$ and $\sigma$ are highly correlated, i.e., $m_{1} \sim 2.2 \mathrm{meV}$ for $(\rho, \sigma) \sim(\pi / 2,0)$ and $m_{1} \sim 6.3 \mathrm{meV}$ for $(\rho, \sigma) \sim(0, \pi / 2)$, as a result of the approximate $\mu-\tau$ reflection symmetry and one-zero texture of $M_{\nu}$.

It is worth remarking that leptoquarks may naturally originate from the grand unification framework, and the leptoquark extensions of the SM can not only give a combined explanation of neutrino masses, lepton flavor mixing and the muon anomalous magnetic moment, as focused on in this work, but also have the potential to address flavor anomalies in the quark sector. Therefore, as appealing and promising extensions of the SM, leptoquark models deserve more attentions and further investigations.

\section{Acknowledgments}

I greatly appreciate the encouragements and useful suggestions from Prof. Zhi-zhong Xing, and also thank Yu-feng Li, Xin Wang and Shun Zhou for helpful discussions. This research work is partly supported by the National Natural Science Foundation of China under grant No. 11775231, No. 11835013 and No. 12075254.

Open Access. This article is distributed under the terms of the Creative Commons Attribution License (CC-BY 4.0), which permits any use, distribution and reproduction in any medium, provided the original author(s) and source are credited.

\section{References}

[1] Z.-z. Xing, Flavor structures of charged fermions and massive neutrinos, Phys. Rept. 854 (2020) 1 [arXiv: 1909.09610] [INSPIRE].

[2] Particle Data Group collaboration, Review of Particle Physics, PTEP 2020 (2020) 083C01 [INSPIRE].

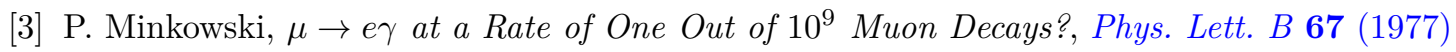
421 [INSPIRE].

[4] T. Yanagida, Horizontal gauge symmetry and masses of neutrinos, Conf. Proc. C $\mathbf{7 9 0 2 1 3 1}$ (1979) 95 [INSPIRE].

[5] M. Gell-Mann, P. Ramond and R. Slansky, Complex Spinors and Unified Theories, Conf. Proc. C $\mathbf{7 9 0 9 2 7}$ (1979) 315 [arXiv: 1306.4669] [InSPIRE]. 
[6] S.L. Glashow, The Future of Elementary Particle Physics, NATO Sci. Ser. B 61 (1980) 687 [INSPIRE].

[7] R.N. Mohapatra and G. Senjanović, Neutrino Mass and Spontaneous Parity Nonconservation, Phys. Rev. Lett. 44 (1980) 912 [InSPIRE].

[8] W. Konetschny and W. Kummer, Nonconservation of Total Lepton Number with Scalar Bosons, Phys. Lett. B 70 (1977) 433 [InSPIRE].

[9] M. Magg and C. Wetterich, Neutrino Mass Problem and Gauge Hierarchy, Phys. Lett. B 94 (1980) 61 [INSPIRE].

[10] J. Schechter and J.W.F. Valle, Neutrino Masses in $\mathrm{SU}(2) \times \mathrm{U}(1)$ Theories, Phys. Rev. D 22 (1980) 2227 [INSPIRE].

[11] T.P. Cheng and L.-F. Li, Neutrino Masses, Mixings and Oscillations in $\mathrm{SU}(2) \times \mathrm{U}(1)$ Models of Electroweak Interactions, Phys. Rev. D 22 (1980) 2860 [InSPIRE].

[12] G. Lazarides, Q. Shafi and C. Wetterich, Proton Lifetime and Fermion Masses in an SO(10) Model, Nucl. Phys. B 181 (1981) 287 [InSPIRE].

[13] R.N. Mohapatra and G. Senjanović, Neutrino Masses and Mixings in Gauge Models with Spontaneous Parity Violation, Phys. Rev. D 23 (1981) 165 [INSPIRE].

[14] R. Foot, H. Lew, X.G. He and G.C. Joshi, Seesaw Neutrino Masses Induced by a Triplet of Leptons, Z. Phys. C 44 (1989) 441 [INSPIRE].

[15] E. Ma, Pathways to naturally small neutrino masses, Phys. Rev. Lett. 81 (1998) 1171 [hep-ph/9805219] [INSPIRE].

[16] A. Zee, A Theory of Lepton Number Violation, Neutrino Majorana Mass, and Oscillation, Phys. Lett. B 93 (1980) 389 [Erratum ibid. 95 (1980) 461] [INSPIRE].

[17] A. Zee, Quantum Numbers of Majorana Neutrino Masses, Nucl. Phys. B 264 (1986) 99 [INSPIRE].

[18] K.S. Babu, Model of 'Calculable' Majorana Neutrino Masses, Phys. Lett. B 203 (1988) 132 [INSPIRE].

[19] Y. Cai, J. Herrero-García, M.A. Schmidt, A. Vicente and R.R. Volkas, From the trees to the forest: a review of radiative neutrino mass models, Front. in Phys. 5 (2017) 63 [arXiv: 1706. 08524] [INSPIRE].

[20] K.S. Babu, P.S.B. Dev, S. Jana and A. Thapa, Non-Standard Interactions in Radiative Neutrino Mass Models, JHEP 03 (2020) 006 [arXiv:1907.09498] [INSPIRE].

[21] Muon $g-2$ collaboration, Measurement of the Positive Muon Anomalous Magnetic Moment to 0.46 ppm, Phys. Rev. Lett. 126 (2021) 141801 [arXiv:2104.03281] [INSPIRE].

[22] Muon $g-2$ collaboration, Final Report of the Muon E821 Anomalous Magnetic Moment Measurement at BNL, Phys. Rev. D 73 (2006) 072003 [hep-ex/0602035] [INSPIRE].

[23] T. Aoyama et al., The anomalous magnetic moment of the muon in the Standard Model, Phys. Rept. 887 (2020) 1 [arXiv: 2006. 04822] [INSPIRE].

[24] S. Borsányi et al., Leading hadronic contribution to the muon magnetic moment from lattice QCD, Nature 593 (2021) 51 [arXiv: 2002.12347] [INSPIRE].

[25] C.-W. Chiang and K. Yagyu, Radiative Seesaw Mechanism for Charged Leptons, Phys. Rev. D 103 (2021) L111302 [arXiv:2104.00890] [INSPIRE]. 
[26] H.M. Lee, Leptoquark option for B-meson anomalies and leptonic signatures, Phys. Rev. D 104 (2021) 015007 [arXiv: 2104.02982] [INSPIRE].

[27] A. Crivellin and M. Hoferichter, Consequences of chirally enhanced explanations of $(g-2)_{\mu}$ for $h \rightarrow \mu \mu$ and $Z \rightarrow \mu \mu$, arXiv:2104.03202 [INSPIRE].

[28] H.-B. Zhang, C.-X. Liu, J.-L. Yang and T.-F. Feng, Muon anomalous magnetic dipole moment in the $\mu \nu S S M$, arXiv:2104.03489 [INSPIRE].

[29] G. Arcadi, L. Calibbi, M. Fedele and F. Mescia, Muon $g-2$ and B-anomalies from Dark Matter, arXiv:2104.03228 [INSPIRE].

[30] B. Zhu and X. Liu, Probing light dark matter with scalar mediator: muon $(g-2)$ deviation, the proton radius puzzle, arXiv:2104.03238 [INSPIRE].

[31] T. Nomura and H. Okada, Explanations for anomalies of muon anomalous magnetic dipole moment, $b \rightarrow s \mu \bar{\mu}$ and radiative neutrino masses in a leptoquark model, arXiv:2104.03248 [INSPIRE].

[32] M. Endo, K. Hamaguchi, S. Iwamoto and T. Kitahara, Supersymmetric Interpretation of the Muon g-2 Anomaly, arXiv:2104.03217 [INSPIRE].

[33] P. Das, M.K. Das and N. Khan, The FIMP-WIMP dark matter and Muon $g-2$ in the extended singlet scalar model, arXiv:2104.03271 [INSPIRE].

[34] S. Baum, M. Carena, N.R. Shah and C.E.M. Wagner, The Tiny $(g-2)$ Muon Wobble from Small- $\mu$ Supersymmetry, arXiv:2104.03302 [INSPIRE].

[35] W. Ahmed, I. Khan, J. Li, T. Li, S. Raza and W. Zhang, The Natural Explanation of the Muon Anomalous Magnetic Moment via the Electroweak Supersymmetry from the GmSUGRA in the MSSM, arXiv:2104.03491 [INSPIRE].

[36] S.-F. Ge, X.-D. Ma and P. Pasquini, Probing the Dark Axion Portal with Muon Anomalous Magnetic Moment, arXiv:2104.03276 [INSPIRE].

[37] X.-F. Han, T. Li, H.-X. Wang, L. Wang and Y. Zhang, Lepton-specific inert two-Higgs-doublet model confronted with the new results for muon and electron $g-2$ anomalies and multi-lepton searches at the LHC, arXiv:2104.03227 [INSPIRE].

[38] Y. Bai and J. Berger, Muon g- 2 in Lepton Portal Dark Matter, arXiv:2104.03301 [INSPIRE].

[39] W.-Y. Keung, D. Marfatia and P.-Y. Tseng, Axion-like particles, two-Higgs-doublet models, leptoquarks, and the electron and muon $g-2$, arXiv:2104.03341 [INSPIRE].

[40] V. Brdar, S. Jana, J. Kubo and M. Lindner, Semi-secretly interacting ALP as an explanation of Fermilab muon $g-2$ measurement, arXiv:2104.03282 [INSPIRE].

[41] L. Zu, X. Pan, L. Feng, Q. Yuan and Y.-Z. Fan, Constraining U(1) $)_{L_{\mu}-L_{\tau}}$ charged dark matter model for muon $g-2$ anomaly with AMS-02 electron and positron data, arXiv:2104.03340 [INSPIRE].

[42] M.A. Buen-Abad, J. Fan, M. Reece and C. Sun, Challenges for an axion explanation of the muon $g-2$ measurement, arXiv:2104.03267 [INSPIRE].

[43] M. Abdughani, Y.-Z. Fan, L. Feng, Y.-L. Sming Tsai, L. Wu and Q. Yuan, A common origin of muon $g-2$ anomaly, Galaxy Center GeV excess and AMS-02 anti-proton excess in the NMSSM, arXiv:2104.03274 [INSPIRE]. 
[44] P.M. Ferreira, B.L. Gonçalves, F.R. Joaquim and M. Sher, $(g-2)_{\mu}$ in the 2HDM and slightly beyond - an updated view, arXiv:2104.03367 [INSPIRE].

[45] M. Ibe, S. Kobayashi, Y. Nakayama and S. Shirai, Muon $g-2$ in Gauge Mediation without SUSY CP Problem, arXiv:2104.03289 [InSPIRE].

[46] K.S. Babu, S. Jana, M. Lindner and V.P. K, Muon g- 2 Anomaly and Neutrino Magnetic Moments, arXiv:2104.03291 [INSPIRE].

[47] M. Van Beekveld, W. Beenakker, M. Schutten and J. De Wit, Dark matter, fine-tuning and $(g-2)_{\mu}$ in the $p M S S M$, arXiv:2104.03245 [INSPIRE].

[48] C.-H. Chen, C.-W. Chiang and T. Nomura, Muon g- 2 in two-Higgs-doublet model with type-II seesaw mechanism, arXiv:2104.03275 [INSPIRE].

[49] P. Cox, C. Han and T.T. Yanagida, Muon g-2 and Co-annihilating Dark Matter in the $M S S M$, arXiv:2104.03290 [INSPIRE].

[50] C. Han, Muon $g-2$ and CP-violation in MSSM, arXiv:2104.03292 [INSPIRE].

[51] M. Cadeddu, N. Cargioli, F. Dordei, C. Giunti and E. Picciau, Muon and electron g-2, proton and cesium weak charges implications on dark $Z_{d}$ models, arXiv:2104.03280 [INSPIRE].

[52] T. Li, J. Pei and W. Zhang, Muon Anomalous Magnetic Moment and Higgs Potential Stability in the 331 Model from $E_{6}$, arXiv:2104.03334 [INSPIRE].

[53] H.-X. Wang, L. Wang and Y. Zhang, muon $g-2$ anomaly and $\mu-\tau$-philic Higgs doublet with a light CP-even component, arXiv:2104.03242 [INSPIRE].

[54] Y. Gu, N. Liu, L. Su and D. Wang, Heavy Bino and Slepton for Muon g- 2 Anomaly, arXiv:2104.03239 [INSPIRE].

[55] F. Wang, L. Wu, Y. Xiao, J.M. Yang and Y. Zhang, GUT-scale constrained SUSY in light of E989 muon $g-2$ measurement, arXiv:2104.03262 [INSPIRE].

[56] L. Calibbi, M.L. López-Ibáñez, A. Melis and O. Vives, Implications of the Muon g-2 result on the flavour structure of the lepton mass matrix, arXiv:2104.03296 [INSPIRE].

[57] W. Yin, Muon g - 2 anomaly in anomaly mediation, JHEP 06 (2021) 029 [arXiv: 2104.03259] [INSPIRE].

[58] J. Cao, J. Lian, Y. Pan, D. Zhang and P. Zhu, Improved $(g-2)_{\mu}$ Measurement and Singlino dark matter in the general NMSSM, arXiv:2104.03284 [INSPIRE].

[59] P. Escribano, J. Terol-Calvo and A. Vicente, $(g-2)_{e, \mu}$ in an extended inverse type-III seesaw model, Phys. Rev. D 103 (2021) 115018 [arXiv:2104.03705] [inSPIRE].

[60] P. Athron, C. Balázs, D.H. Jacob, W. Kotlarski, D. Stöckinger and H. Stöckinger-Kim, New physics explanations of $a_{\mu}$ in light of the FNAL muon $g-2$ measurement, arXiv:2104.03691 [INSPIRE].

[61] J.C. Eung and T. Mondal, Leptophilic bosons and muon $g-2$ at lepton colliders, arXiv:2104.03701 [INSPIRE].

[62] A. Aboubrahim, M. Klasen and P. Nath, What Fermilab $(g-2)_{\mu}$ experiment tells us about discovering SUSY at HL-LHC and HE-LHC, arXiv: 2104.03839 [INSPIRE].

[63] B. Bhattacharya, A. Datta, D. Marfatia, S. Nandi and J. Waite, Axion-like particles resolve the $B \rightarrow \pi K$ and $g-2$ anomalies, arXiv:2104.03947 [INSPIRE]. 
[64] J.-L. Yang, H.-B. Zhang, C.-X. Liu, X.-X. Dong and T.-F. Feng, Muon $(g-2)$ in the $B$-LSSM, arXiv:2104.03542 [INSPIRE].

[65] G. Arcadi, A.S. De Jesus, T.B. De Melo, F.S. Queiroz and Y.S. Villamizar, A $2 H D M$ for the $g-2$ and Dark Matter, arXiv:2104.04456 [INSPIRE].

[66] C.-T. Lu, R. Ramos and Y.-L. Sming Tsai, Shedding light on dark matter with recent muon $(g-2)$ and Higgs exotic decay measurements, arXiv:2104.04503 [INSPIRE].

[67] T. Li, M.A. Schmidt, C.-Y. Yao and M. Yuan, Charged lepton flavor violation in light of the muon magnetic moment anomaly and colliders, arXiv:2104.04494 [INSPIRE].

[68] J.-Y. Cen, Y. Cheng, X.-G. He and J. Sun, Flavor Specific U(1) $B_{B_{q}-L_{\mu}}$ Gauge Model for Muon $g-2$ and $b \rightarrow s \bar{\mu} \mu$ Anomalies, arXiv:2104.05006 [INSPIRE].

[69] D. Marzocca and S. Trifinopoulos, A Minimal Explanation of Flavour Anomalies: B-Meson Decays, Muon Magnetic Moment, and the Cabibbo Angle, arXiv:2104.05730 [INSPIRE].

[70] M. Du, J. Liang, Z. Liu and V.Q. Tran, A vector leptoquark interpretation of the muon $g-2$ and $B$ anomalies, arXiv:2104.05685 [INSPIRE].

[71] S. Zhou, Neutrino Masses, Leptonic Flavor Mixing and Muon $(g-2)$ in the Seesaw Model with the $\mathrm{U}(1)_{L_{\mu}-L_{\tau}}$ Gauge Symmetry, arXiv:2104.06858 [INSPIRE].

[72] K. Ban, Y. Jho, Y. Kwon, S.C. Park, S. Park and P.-Y. Tseng, A comprehensive study of vector leptoquark on the B-meson and Muon $g-2$ anomalies, arXiv:2104.06656 [INSPIRE].

[73] A.E. Cárcamo Hernández, S. Kovalenko, M. Maniatis and I. Schmidt, Fermion mass hierarchy and $g-2$ anomalies in an extended 3HDM Model, arXiv:2104.07047 [INSPIRE].

[74] L.A. Anchordoqui, I. Antoniadis, X. Huang, D. Lüst and T.R. Taylor, Muon $g-2$ discrepancy within D-brane string compactifications, arXiv:2104.06854 [INSPIRE].

[75] H. Baer, V. Barger and H. Serce, Anomalous muon magnetic moment, supersymmetry, naturalness, LHC search limits and the landscape, arXiv:2104.07597 [INSPIRE].

[76] W. Altmannshofer, S.A. Gadam, S. Gori and N. Hamer, Explaining $(g-2)_{\mu}$ with Multi-TeV Sleptons, arXiv:2104.08293 [INSPIRE].

[77] G. Cacciapaglia, C. Cot and F. Sannino, Naturalness of lepton non-universality and muon $g-2, \operatorname{arXiv}: 2104.08818$ [INSPIRE].

[78] A. Dasgupta, S.K. Kang and M. Park, Neutrino mass and $(g-2)_{\mu}$ with dark $\mathrm{U}(1)_{D}$ symmetry, arXiv:2104.09205 [INSPIRE].

[79] A. Aboubrahim, P. Nath and R.M. Syed, Yukawa coupling unification in an $\mathrm{SO}(10)$ model consistent with Fermilab $(g-2)_{\mu}$ result, JHEP 06 (2021) 002 [arXiv:2104.10114] [INSPIRE].

[80] A. Jueid, J. Kim, S. Lee and J. Song, Type-X two Higgs doublet model in light of the muon $g-2$ : confronting Higgs and collider data, arXiv:2104.10175 [INSPIRE].

[81] E. Ma, Gauged lepton number, Dirac neutrinos, dark matter, and muon g-2, Phys. Lett. B 819 (2021) 136402 [arXiv:2104.10324] [INSPIRE].

[82] P.F. Perez, C. Murgui and A.D. Plascencia, Leptoquarks and Matter Unification: Flavor Anomalies and the Muon $g-2$, arXiv:2104.11229 [INSPIRE].

[83] K. Ghorbani, Light vector dark matter with scalar mediator and muon $g-2$ anomaly, arXiv:2104.13810 [INSPIRE]. 
[84] J.A. Carpio, K. Murase, I.M. Shoemaker and Z. Tabrizi, High-energy cosmic neutrinos as a probe of the vector mediator scenario in light of the muon $g-2$ anomaly and Hubble tension, arXiv:2104.15136 [INSPIRE].

[85] B.A. Arbuzov and I.V. Zaitsev, Calculation of the contribution to muon $g-2$ due to the effective anomalous three boson interaction and the new experimental result, arXiv: 2105.00903 [INSPIRE].

[86] J.S. Alvarado, S.F. Mantilla, R. Martinez and F. Ochoa, A non-universal U(1) $X$ extension to the Standard Model to study the B meson anomaly and muon $g-2$, arXiv:2105.04715 [INSPIRE].

[87] W.-F. Chang, One colorful resolution to the neutrino mass generation, three lepton flavor universality anomalies, and the Cabibbo angle anomaly, arXiv:2105.06917 [INSPIRE].

[88] B. Dutta, S. Ghosh, P. Huang and J. Kumar, Explaining $g_{\mu}-2$ and $R_{K^{(*)}}$ using the light mediators of $\mathrm{U}(1)_{T 3 R}$, arXiv:2105.07655 [INSPIRE].

[89] M. Lindner, M. Platscher and F.S. Queiroz, A Call for New Physics: The Muon Anomalous Magnetic Moment and Lepton Flavor Violation, Phys. Rept. 731 (2018) 1

[arXiv:1610.06587] [INSPIRE].

[90] C. Biggio, The Contribution of fermionic seesaws to the anomalous magnetic moment of leptons, Phys. Lett. B 668 (2008) 378 [arXiv:0806.2558] [INSPIRE].

[91] J.C. Pati and A. Salam, Unified Lepton-Hadron Symmetry and a Gauge Theory of the Basic Interactions, Phys. Rev. D 8 (1973) 1240 [InSPIRE].

[92] J.C. Pati and A. Salam, Lepton Number as the Fourth Color, Phys. Rev. D 10 (1974) 275 [Erratum ibid. 11 (1975) 703] [INSPIRE].

[93] H. Fritzsch and P. Minkowski, Unified Interactions of Leptons and Hadrons, Annals Phys. 93 (1975) 193 [INSPIRE].

[94] H. Georgi and S.L. Glashow, Unity of All Elementary Particle Forces, Phys. Rev. Lett. 32 (1974) 438 [inSPIRE].

[95] A. Djouadi, T. Kohler, M. Spira and J. Tutas, (eb), (et) type leptoquarks at ep colliders, Z. Phys. C 46 (1990) 679 [inSPIRE].

[96] S. Davidson, D.C. Bailey and B.A. Campbell, Model independent constraints on leptoquarks from rare processes, Z. Phys. C 61 (1994) 613 [hep-ph/9309310] [InSPIRE].

[97] G. Couture and H. Konig, Bounds on second generation scalar leptoquarks from the anomalous magnetic moment of the muon, Phys. Rev. D 53 (1996) 555 [hep-ph/9507263] [INSPIRE].

[98] M. Hirsch, H.V. Klapdor-Kleingrothaus and S.G. Kovalenko, New low-energy leptoquark interactions, Phys. Lett. B 378 (1996) 17 [hep-ph/9602305] [INSPIRE].

[99] M. Hirsch, H.V. Klapdor-Kleingrothaus and S.G. Kovalenko, New leptoquark mechanism of neutrinoless double beta decay, Phys. Rev. D 54 (1996) R4207 [hep-ph/9603213] [INSPIRE].

[100] C.-K. Chua, X.-G. He and W.-Y.P. Hwang, Neutrino mass induced radiatively by supersymmetric leptoquarks, Phys. Lett. B 479 (2000) 224 [hep-ph/9905340] [INSPIRE].

[101] U. Mahanta, Neutrino masses and mixing angles from leptoquark interactions, Phys. Rev. D 62 (2000) 073009 [hep-ph/9909518] [rNSPIRE]. 
[102] K.-m. Cheung, Muon anomalous magnetic moment and leptoquark solutions, Phys. Rev. D 64 (2001) 033001 [hep-ph/0102238] [INSPIRE].

[103] D. Aristizabal Sierra, M. Hirsch and S.G. Kovalenko, Leptoquarks: Neutrino masses and accelerator phenomenology, Phys. Rev. D 77 (2008) 055011 [arXiv:0710.5699] [INSPIRE].

[104] K.S. Babu and J. Julio, Two-Loop Neutrino Mass Generation through Leptoquarks, Nucl. Phys. B 841 (2010) 130 [arXiv:1006.1092] [INSPIRE].

[105] H. Päs and E. Schumacher, Common origin of $R_{K}$ and neutrino masses, Phys. Rev. D 92 (2015) 114025 [arXiv:1510.08757] [INSPIRE].

[106] M. Bauer and M. Neubert, Minimal Leptoquark Explanation for the $R_{D^{(*)}}, R_{K}$, and $(g-2)_{\mu}$ Anomalies, Phys. Rev. Lett. 116 (2016) 141802 [arXiv:1511.01900] [INSPIRE].

[107] K. Cheung, T. Nomura and H. Okada, Testable radiative neutrino mass model without additional symmetries and explanation for the $b \rightarrow s \ell^{+} \ell^{-}$anomaly, Phys. Rev. D 94 (2016) 115024 [arXiv: 1610.02322] [INSPIRE].

[108] E. Coluccio Leskow, G. D'Ambrosio, A. Crivellin and D. Müller, $(g-2) \mu$, lepton flavor violation, and $Z$ decays with leptoquarks: Correlations and future prospects, Phys. Rev. D 95 (2017) 055018 [arXiv: 1612.06858] [INSPIRE].

[109] I. Doršner, S. Fajfer and N. Košnik, Leptoquark mechanism of neutrino masses within the grand unification framework, Eur. Phys. J. C 77 (2017) 417 [arXiv:1701. 08322] [InSPIRE].

[110] Y. Cai, J. Gargalionis, M.A. Schmidt and R.R. Volkas, Reconsidering the One Leptoquark solution: flavor anomalies and neutrino mass, JHEP 10 (2017) 047 [arXiv:1704.05849] [INSPIRE].

[111] A. Angelescu, D. Bečirević, D.A. Faroughy and O. Sumensari, Closing the window on single leptoquark solutions to the B-physics anomalies, JHEP 10 (2018) 183 [arXiv:1808.08179] [INSPIRE].

[112] I. Bigaran, J. Gargalionis and R.R. Volkas, A near-minimal leptoquark model for reconciling flavour anomalies and generating radiative neutrino masses, JHEP 10 (2019) 106 [arXiv: 1906.01870] [INSPIRE].

[113] I. Doršner, S. Fajfer and O. Sumensari, Muon g- 2 and scalar leptoquark mixing, JHEP 06 (2020) 089 [arXiv: 1910.03877] [inSPIRE].

[114] S. Saad and A. Thapa, Common origin of neutrino masses and $R_{D^{(*)}}, R_{K^{(*)}}$ anomalies, Phys. Rev. D 102 (2020) 015014 [arXiv:2004.07880] [INSPIRE].

[115] P.S. Bhupal Dev, R. Mohanta, S. Patra and S. Sahoo, Unified explanation of flavor anomalies, radiative neutrino masses, and ANITA anomalous events in a vector leptoquark model, Phys. Rev. D 102 (2020) 095012 [arXiv:2004.09464] [INSPIRE].

[116] S. Saad, Combined explanations of $(g-2)_{\mu}, R_{D^{(*)}}, R_{K^{(*)}}$ anomalies in a two-loop radiative neutrino mass model, Phys. Rev. D 102 (2020) 015019 [arXiv:2005.04352] [INSPIRE].

[117] I. Doršner, S. Fajfer and S. Saad, $\mu \rightarrow e \gamma$ selecting scalar leptoquark solutions for the $(g-2)_{e, \mu}$ puzzles, Phys. Rev. D 102 (2020) 075007 [arXiv:2006.11624] [INSPIRE].

[118] A. Crivellin, D. Mueller and F. Saturnino, Correlating $h \rightarrow \mu^{+} \mu^{-}$to the Anomalous Magnetic Moment of the Muon via Leptoquarks, arXiv:2008.02643 [INSPIRE].

[119] V. Gherardi, D. Marzocca and E. Venturini, Low-energy phenomenology of scalar leptoquarks at one-loop accuracy, JHEP 01 (2021) 138 [arXiv:2008.09548] [INSPIRE]. 
[120] K.S. Babu, P.S.B. Dev, S. Jana and A. Thapa, Unified framework for B-anomalies, muon $g-2$ and neutrino masses, JHEP 03 (2021) 179 [arXiv:2009.01771] [INSPIRE].

[121] A. Crivellin, C. Greub, D. Müller and F. Saturnino, Scalar Leptoquarks in Leptonic Processes, JHEP 02 (2021) 182 [arXiv:2010.06593] [INSPIRE].

[122] I. Doršner, S. Fajfer, A. Greljo, J.F. Kamenik and N. Košnik, Physics of leptoquarks in precision experiments and at particle colliders, Phys. Rept. 641 (2016) 1

[arXiv: 1603.04993] [INSPIRE].

[123] P.F. Harrison and W.G. Scott, $\mu-\tau$ reflection symmetry in lepton mixing and neutrino oscillations, Phys. Lett. B 547 (2002) 219 [hep-ph/0210197] [INSPIRE].

[124] Z.-z. Xing and Z.-h. Zhao, A review of $\mu-\tau$ flavor symmetry in neutrino physics, Rept. Prog. Phys. 79 (2016) 076201 [arXiv: 1512.04207] [INSPIRE].

[125] J.M. Arnold, B. Fornal and M.B. Wise, Phenomenology of scalar leptoquarks, Phys. Rev. D 88 (2013) 035009 [arXiv:1304.6119] [INSPIRE].

[126] C. Murgui and M.B. Wise, Scalar Leptoquarks, Baryon Number Violation and Pati-Salam Symmetry, arXiv:2105.14029 [INSPIRE].

[127] A. Denner, H. Eck, O. Hahn and J. Kublbeck, Compact Feynman rules for Majorana fermions, Phys. Lett. B 291 (1992) 278 [INSPIRE].

[128] A. Denner, H. Eck, O. Hahn and J. Kublbeck, Feynman rules for fermion number violating interactions, Nucl. Phys. B 387 (1992) 467 [INSPIRE].

[129] D. Binosi and L. Theussl, JaxoDraw: A Graphical user interface for drawing Feynman diagrams, Comput. Phys. Commun. 161 (2004) 76 [hep-ph/0309015] [INSPIRE].

[130] B.A. Kniehl and A. Pilaftsis, Mixing renormalization in Majorana neutrino theories, Nucl. Phys. B 474 (1996) 286 [hep-ph/9601390] [InSPIRE].

[131] H.H. Patel, Package-X: A Mathematica package for the analytic calculation of one-loop integrals, Comput. Phys. Commun. 197 (2015) 276 [arXiv:1503.01469] [INSPIRE].

[132] H.H. Patel, Package-X 2.0: A Mathematica package for the analytic calculation of one-loop integrals, Comput. Phys. Commun. 218 (2017) 66 [arXiv:1612.00009] [INSPIRE].

[133] B. Pontecorvo, Mesonium and anti-mesonium, Sov. Phys. JETP 6 (1957) 429 [inSPIRE].

[134] Z. Maki, M. Nakagawa and S. Sakata, Remarks on the unified model of elementary particles, Prog. Theor. Phys. 28 (1962) 870 [INSPIRE].

[135] B. Pontecorvo, Neutrino Experiments and the Problem of Conservation of Leptonic Charge, Zh. Eksp. Teor. Fiz. 53 (1967) 1717 [INSPIRE].

[136] N. Nath, Z.-z. Xing and J. Zhang, $\mu-\tau$ Reflection Symmetry Embedded in Minimal Seesaw, Eur. Phys. J. C 78 (2018) 289 [arXiv:1801.09931] [INSPIRE].

[137] Z.-Z. Xing and D. Zhang, Seesaw mirroring between light and heavy Majorana neutrinos with the help of the $S_{3}$ reflection symmetry, JHEP 03 (2019) 184 [arXiv:1901.07912] [INSPIRE].

[138] I. Aizawa and M. Yasue, General property of neutrino mass matrix and CP-violation, Phys. Lett. B 607 (2005) 267 [hep-ph/0409331] [INSPIRE].

[139] T. Baba and M. Yasue, Majorana CP-violation in Approximately $\mu-\tau$ Symmetric Models with $\operatorname{det}\left(M_{\nu}\right)=0$, Prog. Theor. Phys. 123 (2010) 659 [arXiv:1003.1438] [INSPIRE]. 
[140] Z.-z. Xing and Y.-L. Zhou, A Generic Diagonalization of the $3 \times 3$ Neutrino Mass Matrix and Its Implications on the $\mu-\tau$ Flavor Symmetry and Maximal CP-violation, Phys. Lett. $B 693$ (2010) 584 [arXiv: 1008.4906] [INSPIRE].

[141] D. Zhang, Integral solutions to the one-loop renormalization-group equations for lepton flavor mixing parameters and the Jarlskog invariant, Nucl. Phys. B 961 (2020) 115260 [arXiv: 2007.12976] [INSPIRE].

[142] G.-y. Huang and S. Zhou, Precise Values of Running Quark and Lepton Masses in the Standard Model, Phys. Rev. D 103 (2021) 016010 [arXiv: 2009.04851] [InSPIRE].

[143] I. Esteban, M.C. Gonzalez-Garcia, M. Maltoni, T. Schwetz and A. Zhou, The fate of hints: updated global analysis of three-flavor neutrino oscillations, JHEP 09 (2020) 178 [arXiv: 2007.14792] [INSPIRE]. 